\title{
Schwartz functions on Nash manifolds
}

\author{
Avraham Aizenbud and Dmitry Gourevitch *
}

July 11, 2011

\begin{abstract}
In this paper we extend the notions of Schwartz functions, tempered functions and generalized Schwartz functions to Nash (i.e. smooth semi-algebraic) manifolds. We reprove for this case classically known properties of Schwartz functions on $\mathbb{R}^{n}$ and build some additional tools which are important in representation theory.
\end{abstract}

\section{Contents}

1 Introduction 2

1.1 Main results . . . . . . . . . . . . . . . . . . . . 3

1.2 Schwartz sections of Nash bundles . . . . . . . . . . . . . . . . . . 4

1.3 Restricted topology and sheaf properties . . . . . . . . . . . . 4

1.4 Possible applications . . . . . . . . . . . . . . . . 5

1.5 Summary . . . . . . . . . . . . . . . . . 6

1.6 Remarks . . . . . . . . . . . . . . . . . . . 6 6

2 Semi-algebraic geometry 8

2.1 Basic notions . . . . . . . . . . . . . . . . 8

2.2 Tarski-Seidenberg principle of quantifier elimination

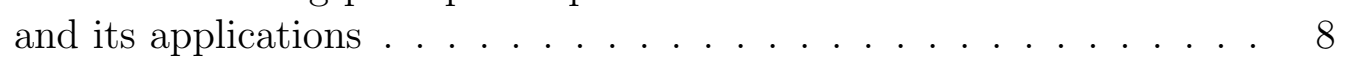

2.3 Additional preliminary results . . . . . . . . . . . . . . . 10

${ }^{*}$ Avraham Aizenbud and Dmitry Gourevitch, Faculty of Mathematics and Computer Science, The Weizmann Institute of Science POB 26, Rehovot 76100, ISRAEL. E-mails: aizenr@yahoo.com, guredim@yahoo.com.

Keywords: Schwartz functions, tempered functions, generalized functions, distributions, Nash manifolds. 
3 Nash manifolds 11

3.1 Nash submanifolds of $\mathbb{R}^{n} \ldots \ldots \ldots \ldots \ldots$. . . . . . . . . 11

3.2 Restricted topological spaces and sheaf theory over them. . . . . . . . 12

3.3 Abstract Nash manifolds . . . . . . . . . . . . . . . . . . . . . . 14

3.3.1 Examples and Remarks . . . . . . . . . . . . . . . . 14

3.4 Nash vector bundles . . . . . . . . . . . . . . . . . 15

3.5 Nash differential operators . . . . . . . . . . . . . . 16

3.5.1 Algebraic differential operators on a Nash manifold . . . . . . 17

3.6 Nash tubular neighborhood . . . . . . . . . . . . . 18

4 Schwartz and tempered functions on affine Nash manifolds 19

4.1 Schwartz functions . . . . . . . . . . . . . . . 19

4.2 Tempered functions . . . . . . . . . . . . . . . 20

4.3 Extension by zero of Schwartz functions . . . . . . . . . . . . . 20

4.4 Partition of unity . . . . . . . . . . . . . . . . 21

4.5 Restriction and sheaf property of tempered functions . . . . . . . . 22

4.6 Extension of Schwartz and tempered functions from closed submanifolds 23

5 Schwartz, tempered and generalized Schwartz sections of Nash bundles over arbitrary Nash manifolds 24

5.1 Main definitions . . . . . . . . . . . . . . . . 24

5.2 Partition of unity . . . . . . . . . . . . . 25

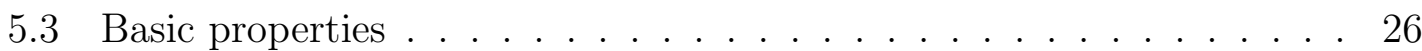

5.4 Characterization of Schwartz functions on open subset . . . . . . 27

5.4 Remarks . . . . . . . . . . . . . . . . 28

5.5 Generalized Schwartz sections supported on closed submanifolds . . . 29

A Appendix $\quad 30$

A.1 Nash structures on standard bundles . . . . . . . . . . . . . . 30

A.1.1 The bundle of densities $D_{M}$. . . . . . . . . . . . . . . . . . . 30

A.2 Semi-algebraic notions on Nash manifolds . . . . . . . . . . . 31

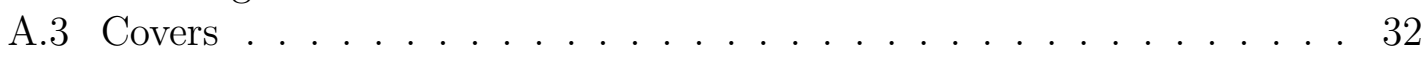

A.4 Cosheaves over restricted topological spaces . . . . . . . . . . . 34

\section{Introduction}

Let us start with the following motivating example. Consider the circle $S^{1}$, let $N \subset S^{1}$ be the north pole and denote $U:=S^{1} \backslash N$. Note that $U$ is diffeomorphic to $\mathbb{R}$ via the stereographic projection. Consider the space $\mathcal{D}\left(S^{1}\right)$ of distributions on $S^{1}$, that is the space of continuous linear functionals on the Fréchet space $C^{\infty}\left(S^{1}\right)$. Consider the subspace $\mathcal{D}_{S^{1}}(N) \subset \mathcal{D}\left(S^{1}\right)$ consisting of all distributions supported 
at $N$. Then the quotient $\mathcal{D}\left(S^{1}\right) / \mathcal{D}_{S^{1}}(N)$ will not be the space of distributions on $U$. However, it will be the space $\mathcal{S}^{*}(U)$ of Schwartz distributions on $U$, that is continuous functionals on the Fréchet space $\mathcal{S}(U)$ of Schwartz functions on $U$. In this case, $\mathcal{S}(U)$ can be identified with $\mathcal{S}(\mathbb{R})$ via the stereographic projection.

The space of Schwartz functions on $\mathbb{R}$ is defined to be the space of all infinitely differentiable functions that rapidly decay at infinity together with all their derivatives, i.e. $x^{n} f^{(k)}$ is bounded for any $n, k$.

The goal of this paper is to extend the notions of Schwartz functions and Schwartz distributions to a larger geometric realm.

As we can see, the definition is of algebraic nature. Hence it would not be reasonable to try to extend it to arbitrary smooth manifolds. However, it is reasonable to extend this notion to smooth algebraic varieties. Unfortunately, sometimes this is not enough. For example, a connected component of real algebraic variety is not always an algebraic variety. By this reason we extend this notion to smooth semi-algebraic manifolds. They are called Nash manifolds! 1 .

For any Nash manifold $M$, we will define the spaces $\mathcal{G}(M), \mathcal{T}(M)$ and $\mathcal{S}(M)$ of generalized Schwartz functions 2 , tempered functions and Schwartz functions on $M$. Informally, $\mathcal{T}(M)$ is the ring of functions that have no more than polynomial growth together with all their derivatives, $\mathcal{G}(M)$ is the space of generalized functions with no more than polynomial growth and $\mathcal{S}(M)$ is the space of functions that decay together with all their derivatives faster than any inverse power of a polynomial.

As in the classical case, in order to define generalized Schwartz functions, we have to define Schwartz functions first. Both $\mathcal{G}(M)$ and $\mathcal{S}(M)$ are modules over $\mathcal{T}(M)$.

The triple $\mathcal{S}(M), \mathcal{T}(M), \mathcal{G}(M)$ is analogous to $C_{c}^{\infty}(M), C^{\infty}(M)$ and $C^{-\infty}(M)$ but it has additional nice properties as we will see later.

We will show that for $M=\mathbb{R}^{n}, \mathcal{S}(M)$ is the space of classical Schwartz functions and $\mathcal{G}(M)$ is the space of classical generalized Schwartz functions. For compact Nash manifold $M, \mathcal{S}(M)=\mathcal{T}(M)=C^{\infty}(M)$.

\subsection{Main results}

In this subsection we summarize the main results of the paper.

Result 1.1.1 Let $M$ be a Nash manifold and $Z \subset M$ be a closed Nash submanifold.

\footnotetext{
${ }^{1}$ The necessary preliminaries on Nash manifolds are given in sections 2 and 3 .

${ }^{2}$ In this paper we distinguish between the (similar) notions of a generalized function and a distribution. They can be identified by choosing a measure. Without fixing a measure, a smooth function defines a generalized function but not a distribution. We will discuss it later in more details.
} 
Then the restriction maps $\mathcal{T}(M) \rightarrow \mathcal{T}(Z)$ and $\mathcal{S}(M) \rightarrow \mathcal{S}(Z)$ are onto (see theorems 4.6.1 and 4.6.2).

Result 1.1.2 Let $M$ be a Nash manifold and $U \subset M$ be a semi-algebraic open subset. Then a Schwartz function on $U$ is the same as a Schwartz function on $M$ which vanishes with all its derivatives on $M \backslash U$ (see theorem 5.4.3).

This theorem tells us that extension by zero $\mathcal{S}(U) \rightarrow \mathcal{S}(M)$ is a closed imbedding, and hence restriction morphism $\mathcal{G}(M) \rightarrow \mathcal{G}(U)$ is onto.

Classical generalized functions do not have this property. This was our main motivation for extending the definition of Schwartz functions.

\subsection{Schwartz sections of Nash bundles}

Similar notions will be defined for Nash bundles, i.e. smooth semi-algebraic bundles.

For any Nash bundle $E$ over $M$ we will define the spaces $\mathcal{G}(M, E), \mathcal{T}(M, E)$ and $\mathcal{S}(M, E)$ of generalized Schwartz, tempered and Schwartz sections of $E$.

As in the classical case, a generalized Schwartz function is not exactly a functional on the space of Schwartz functions, but a functional on Schwartz densities, i.e. Schwartz sections of the bundle of densities.

Therefore, we will define generalized Schwartz sections by $\mathcal{G}(M, E)=$ $(\mathcal{S}(M, \widetilde{E}))^{*}$, where $\widetilde{E}=E^{*} \otimes D_{M}$ and $D_{M}$ is the bundle of densities on M.

Let $Z \subset M$ be a closed Nash submanifold, and $U=M \backslash Z$. Result 1.1.2 tells us that the quotient space of $\mathcal{G}(M)$ by the subspace $\mathcal{G}(M)_{Z}$ of generalized Schwartz functions supported in $Z$ is $\mathcal{G}(U)$. Hence it is useful to study the space $\mathcal{G}(M)_{Z}$. As in the classical case, $\mathcal{G}(M)_{Z}$ has a filtration by the degree of transversal derivatives of delta functions. The quotients of the filtration are generalized Schwartz sections over $Z$ of symmetric powers of normal bundle to $Z$ in $M$, after a twist.

This result can be extended to generalized Schwartz sections of arbitrary Nash bundles (see corollary 5.5.4).

\subsection{Restricted topology and sheaf properties}

Similarly to algebraic geometry, the reasonable topology on Nash manifolds to consider is a topology in which open sets are open semi-algebraic sets. Unfortunately, it is not a topology in the usual sense of the word, it is only what is called restricted topology. This means that the union of an infinite number of open sets does not have to be open. The only open covers considered in the restricted topology are finite open covers.

The restriction of a generalized Schwartz function (respectively of a tempered function) to an open subset is again a generalized Schwartz (respectively a tempered 
function). This means that they form pre-sheaves. We will show that they are actually sheaves, which means that for any finite open cover $M=\bigcup_{i=1}^{n} U_{i}$, a function $\alpha$ on $M$ is tempered if and only if $\left.\alpha\right|_{U_{i}}$ is tempered for all $i$. It is of course not true for infinite covers. For definitions of a pre-sheaf and a sheaf in the restricted topology see section 3.2. We denote the sheaf of generalized Schwartz functions by $\mathcal{G}_{M}$ and the sheaf of tempered functions by $\mathcal{T}_{M}$. By result 1.1.2, $\mathcal{G}_{M}$ is a flabby sheaf.

Similarly, for any Nash bundle $E$ over $M$ we will define the sheaf $\mathcal{T}_{M}^{E}$ of tempered sections and the sheaf $\mathcal{G}_{M}^{E}$ of generalized Schwartz sections.

As we have mentioned before, Schwartz functions behave similarly to compactly supported smooth functions. In particular, they cannot be restricted to an open subset, but can be extended by zero from an open subset. This means that they do not form a sheaf, but an object dual to a sheaf, a so-called cosheaf. The exact definition of a cosheaf will be given in the appendix (section A.4). We denote the cosheaf of Schwartz functions by $\mathcal{S}_{M}$. We will prove that $\mathcal{S}_{M}$ is actually a cosheaf and not just pre-cosheaf by proving a Schwartz version of the partition of unity theorem. Similarly, for any Nash bundle $E$ over $M$ we will define the cosheaf $\mathcal{S}_{M}^{E}$ of Schwartz sections.

\subsection{Possible applications}

Schwartz functions are used in the representation theory of algebraic groups. Our definition coincides with Casselman's definition (cf. [Cas1]) for algebraic groups. Our paper allows to use Schwartz functions in additional situations in the representation theory of algebraic groups, since an orbit of an algebraic action is a Nash manifold, but does not have to be an algebraic group or even an algebraic variety.

Generalized Schwartz sections can be used for "devisage". We mean the following. Let $U \subset M$ be an open (semi-algebraic) subset. Instead of dealing with generalized Schwartz sections of a bundle on $M$, we can deal with generalized Schwartz sections of its restriction to $U$ and generalized Schwartz sections of some other bundles on $M \backslash U$ (see 5.5.4).

For example if we are given an action of an algebraic group $G$ on an algebraic variety $M$, and a $G$-equivariant bundle $E$ over $M$, then devisage to orbits helps us to investigate the space of $G$-invariant generalized sections of $E$. One of the implementations of this argument appears in [AGS]. There we also use the fact that $\mathcal{S}\left(\mathbb{R}^{n}\right)$ is preserved by Fourier transform. 


\subsection{Summary}

To sum up, for any Nash manifold $M$ we define a sheaf $\mathcal{T}_{M}$ of algebras on $M$ (in the restricted topology) consisting of tempered functions, a sheaf $\mathcal{G}_{M}$ of modules over $\mathcal{T}_{M}$ consisting of generalized Schwartz functions, and a cosheaf $\mathcal{S}_{M}$ of modules over $\mathcal{T}_{M}$ consisting of Schwartz functions.

Moreover, for any Nash bundle $E$ over $M$ we define sheaves $\mathcal{T}_{M}^{E}$ and $\mathcal{G}_{M}^{E}$ of modules over $\mathcal{T}_{M}$ consisting of tempered and generalized Schwartz sections of $E$ respectively and a cosheaf $\mathcal{S}_{M}^{E}$ of modules over $\mathcal{T}_{M}$ consisting of Schwartz sections of $E$.

Let us list the main properties of these objects that we will prove in this paper:

1. Compatibility: For open semi-algebraic subset $U \subset M,\left.\mathcal{S}_{M}^{E}\right|_{U}=\mathcal{S}_{U}^{\left.E\right|_{U}},\left.\mathcal{T}_{M}^{E}\right|_{U}=$ $\mathcal{T}_{U}^{\left.E\right|_{U}},\left.\mathcal{G}_{M}^{E}\right|_{U}=\mathcal{G}_{U}^{\left.E\right|_{U}}$

2. $\mathcal{S}\left(\mathbb{R}^{n}\right)=$ Classical Schwartz functions on $\mathbb{R}^{n}$ (see corollary 4.1.3).

3. For compact $M, \mathcal{S}(M, E)=\mathcal{T}(M, E)=C^{\infty}(M, E)$ (see theorem 5.3.1).

4. $\mathcal{G}_{M}^{E}=\left(\mathcal{S}_{M}^{\widetilde{E}}\right)^{*}$, where $\widetilde{E}=E^{*} \otimes D_{M}$ and $D_{M}$ is the bundle of densities on $M$.

5. Let $Z \subset M$ be a closed Nash submanifold. Then the restriction maps $\mathcal{S}(M, E)$ onto $\mathcal{S}\left(Z,\left.E\right|_{Z}\right)$ and $\mathcal{T}(M, E)$ onto $\mathcal{T}\left(Z,\left.E\right|_{Z}\right)$ (see section 5.3$)$.

6. Let $U \subset M$ be a semi-algebraic open subset, then

$$
\mathcal{S}_{M}^{E}(U) \cong\{\phi \in \mathcal{S}(M, E) \mid \quad \phi \text { is } 0 \text { on } M \backslash U \text { with all derivatives }\}
$$

(see theorem 5.4.3).

7. Let $Z \subset M$ be a closed Nash submanifold. Consider $\mathcal{G}(M, E)_{Z}=\{\xi \in$ $\mathcal{G}(M, E) \mid \xi$ is supported in $Z\}$. It has a canonical filtration such that its factors are canonically isomorphic to $\mathcal{G}\left(Z,\left.\left.E\right|_{Z} \otimes S^{i}\left(N_{Z}^{M}\right) \otimes D_{M}^{*}\right|_{Z} \otimes D_{Z}\right)$ where $N_{Z}^{M}$ is the normal bundle of $Z$ in $M$ and $S^{i}$ means $i$-th symmetric power (see corollary 5.5.4).

\subsection{Remarks}

Remark 1.6.1 Harish-Chandra has defined a Schwartz space for every reductive Lie group. However, Harish-Chandra's Schwartz space does not coincide with the space of Schwartz functions that we define in this paper even for the algebraic group $\mathbb{R}^{\times}$. 
Remark 1.6.2 There is a different approach to the concept of Schwartz functions. Namely, if $M$ is embedded as an open subset in a compact manifold $K$ then one can define the space of Schwartz functions on $M$ to be the space of all smooth functions on $K$ that vanish outside $M$ together with all their derivatives. This approach is implemented in [CHM], [KS], [Mor] and [Pre]. In general, this definition depends on the embedding into $K$. Our results show that for Nash manifolds $M$ and $K$ it coincides with our definition and hence does not depend on the embedding.

Remark 1.6.3 After the completion of this project we found out that many of the properties of Schwartz functions on affine Nash manifolds, that is most of section 4 have been obtained already in [dCl].

Remark 1.6.4 In fact, tempered functions can be defined in terms of Schwartz functions or in terms of generalized Schwartz functions by

$$
\begin{aligned}
\mathcal{T}(M, E) & =\left\{\alpha \in C^{\infty}(M, E) \mid \alpha \mathcal{S}(M) \subset \mathcal{S}(M, E)\right\}= \\
& =\left\{\alpha \in C^{\infty}(M, E) \mid \alpha \mathcal{G}(M) \subset \mathcal{G}(M, E)\right\},
\end{aligned}
$$

but the proof is rather technical and lies out of the scope of this paper.

Remark 1.6.5 Throughout the whole paper "smooth" means infinitely differentiable and "bounded" means bounded in absolute value, unless stated otherwise.

\section{Acknowledgements}

We would like to thank our teacher Prof. Joseph Bernstein for teaching us most of the mathematics we know and for his help in this work.

We would also like to thank Prof. William Casselman who dealt with similar things in the early 90 s and sent us his unpublished material.

We thank Prof. Michel Duflo for drawing our attention to the work [dCl] and Prof. Pierre Schapira for drawing our attention to the works [KS], [Mor] and [Pre] which are related to this paper.

We would like to thank Prof. Semyon Alesker, Lev Buhovski, Prof. Alexander Braverman, Vadim Kosoy, Prof. Pierre Milman, Prof. Vitali Milman, Dr. Omer Offen, Dr. Eitan Sayag, Dr. Michael Temkin and Dr. Ilya Tyomkin for helpful discussions.

Also we would like to thank Dr. Ben-Zion Aizenbud, Shifra Reif, Ilya Surdin and Frol Zapolsky for proof reading.

Finally, we would like to thank Prof. Paul Biran, Prof. Vladimir Berkovich, Prof. Stephen Gelbart, Prof. Eugenii Shustin, Prof. Sergei Yakovenko for their useful remarks. 


\section{Semi-algebraic geometry}

In this section we give some preliminaries on semi-algebraic geometry from [BCR].

\subsection{Basic notions}

Definition 2.1.1 $A$ subset $A \subset \mathbb{R}^{n}$ is called a semi-algebraic set iff it can be presented as a finite union of sets defined by a finite number of polynomial equalities and inequalities. In other words, there exist finitely many polynomials $f_{i j}, g_{i k} \in$ $\mathbb{R}\left[x_{1}, \ldots, x_{n}\right]$ such that

$$
A=\bigcup_{i=1}^{r}\left\{x \in \mathbb{R}^{n} \mid f_{i 1}(x)>0, \ldots, f_{i s_{i}}(x)>0, g_{i 1}(x)=0, \ldots, g_{i t_{i}}(x)=0\right\} \text {. }
$$

Lemma 2.1.1 The collection of semi-algebraic sets is closed with respect to finite unions, finite intersections, and complements.

The proof is immediate.

Definition 2.1.2 Let $A \subset \mathbb{R}^{n}$ and $B \subset \mathbb{R}^{m}$ be semi-algebraic sets. $A$ mapping $\nu: A \rightarrow B$ is called semi-algebraic iff its graph is a semi-algebraic subset of $\mathbb{R}^{m+n}$.

\subsection{Tarski-Seidenberg principle of quantifier elimination and its applications}

One of the main tools in the theory of semi-algebraic spaces is the Tarski-Seidenberg principle of quantifier elimination. Here we will formulate and use a special case of it. We start from the geometric formulation.

Theorem 2.2.1 Let $A \subset \mathbb{R}^{n}$ be a semi-algebraic subset and $p: \mathbb{R}^{n} \rightarrow \mathbb{R}^{n-1}$ be the standard projection. Then the image $p(A)$ is a semi-algebraic subset of $\mathbb{R}^{n-1}$.

By induction and a standard graph argument we get the following corollary.

Corollary 2.2.2 An image of a semi-algebraic subset of $\mathbb{R}^{n}$ under a semi-algebraic map is semi-algebraic.

Sometimes it is more convenient to use the logical formulation of the TarskiSeidenberg principle. Informally it says that any set that can be described in semialgebraic language is semi-algebraic. We will now give the logical formulation and immediately after that define the logical notion used in it. 
Theorem 2.2.3 (Tarski-Seidenberg principle) Let $\Phi$ be a formula of the language $L(\mathbb{R})$ of ordered fields with parameters in $\mathbb{R}$. Then there exists a quantifier - free formula $\Psi$ of $L(\mathbb{R})$ with the same free variables $x_{1}, \ldots, x_{n}$ as $\Phi$ such that $\forall x \in$ $\mathbb{R}^{n}, \Phi(x) \Leftrightarrow \Psi(x)$

For the proof see Proposition 2.2.4 on page 28 of [BCR.

Definition 2.2.1 A first-order formula of the language of ordered fields with parameters in $\mathbb{R}$ is a formula written with a finite number of conjunctions, disjunctions, negations and universal and existential quantifiers $(\forall$ and $\exists$ ) on variables, starting from atomic formulas which are formulas of the kind $f\left(x_{1}, \ldots, x_{n}\right)=0$ or $g\left(x_{1}, \ldots, x_{n}\right)>0$, where $f$ and $g$ are polynomials with coefficients in $\mathbb{R}$. The free variables of a formula are those variables of the polynomials which are not quantified. We denote the language of such formulas by $L(\mathbb{R})$.

Notation 2.2.2 Let $\Phi$ be a formula of $L(\mathbb{R})$ with free variables $x_{1}, \ldots, x_{n}$. It defines the set of all points $\left(x_{1}, \ldots, x_{n}\right)$ in $\mathbb{R}^{n}$ that satisfy $\Phi$. We denote this set by $\mathbf{S}_{\boldsymbol{\Phi}}$. In short,

$$
\mathbf{S}_{\boldsymbol{\Phi}}:=\left\{x \in \mathbb{R}^{n} \mid \Phi(x)\right\}
$$

Corollary 2.2.4 Let $\Phi$ be a formula of $L(\mathbb{R})$. Then $S_{\Phi}$ is a semi-algebraic set.

Proof. Let $\Psi$ be a quantifier-free formula equivalent to $\Phi$. The set $S_{\Psi}$ is semialgebraic since it is a finite union of sets defined by polynomial equalities and inequalities. Hence $S_{\Phi}$ is also semi-algebraic since $S_{\Phi}=S_{\Psi}$.

Proposition 2.2.5 The logical formulation of the Tarski-Seidenberg principle implies the geometric one.

Proof. Let $A \subset \mathbb{R}^{n}$ be a semi-algebraic subset, and $p r: \mathbb{R}^{n} \rightarrow \mathbb{R}^{n-1}$ the standard projection. Then there exists a formula $\Phi \in L(\mathbb{R})$ such that $A=S_{\Phi}$. Then $\operatorname{pr}(A)=$ $S_{\Psi}$ where

$$
\Psi(y)=“ \exists x \in \mathbb{R}^{n}(\operatorname{pr}(x)=y \wedge \Phi(x)) " .
$$

Since $\Psi \in L(\mathbb{R})$, the claim follows from the previous corollary.

Remark 2.2.6 In fact, it is not difficult to deduce the logical formulation from the geometric one.

Let us now demonstrate how to use the logical formulation of the Tarski-Seidenberg principle.

Corollary 2.2.7 The closure of a semi-algebraic set is semi-algebraic. 
Proof. Let $A \subset \mathbb{R}^{n}$ be a semi-algebraic subset, and let $\bar{A}$ be its closure. Then $\bar{A}=S_{\Psi}$ where

$$
\Psi(x)=" \forall \varepsilon>0 \exists y \in A|x-y|^{2}<\varepsilon " .
$$

Clearly, $\Psi \in L(\mathbb{R})$ and hence $\bar{A}$ is semi-algebraic.

Corollary 2.2.8 Images and preimages of semi-algebraic sets under semi-algebraic mappings are semi-algebraic.

Proposition 2.2.9 Let $\nu$ be a bijective semi-algebraic mapping. Then the inverse mapping $\nu^{-1}$ is also semi-algebraic.

Proof. The graph of $\nu$ is obtained from the graph of $\nu^{-1}$ by switching the coordinates.

\section{Proposition 2.2.10}

(i) The composition of semi-algebraic mappings is semi-algebraic.

(ii) The $\mathbb{R}$-valued semi-algebraic functions on a semi-algebraic set $A$ form a ring, and any nowhere vanishing semi-algebraic function is invertible in this ring.

Proof.

(i) Let $\mu: A \rightarrow B$ and $\nu: B \rightarrow C$ be semi-algebraic mappings. Let $\Gamma_{\mu} \subset \mathbb{R}^{m+n}$ be the graph of $\mu$ and $\Gamma_{\nu} \subset \mathbb{R}^{n+p}$ be the graph of $\nu$. The graph of $\nu \circ \mu$ is the projection of $\left(\Gamma_{\mu} \times \mathbb{R}^{p}\right) \cap\left(\mathbb{R}^{m} \times \Gamma_{\nu}\right)$ onto $\mathbb{R}^{m+p}$ and hence is semi-algebraic.

(ii) follows from (i) by noting that $F+G$ is the composition of $(F, G): A \rightarrow \mathbb{R}^{2}$ with $+: \mathbb{R}^{2} \rightarrow \mathbb{R}, F G$ is the composition of $(F, G): A \rightarrow \mathbb{R}^{2}$ with $\times: \mathbb{R}^{2} \rightarrow \mathbb{R}$ and $\frac{1}{F}$ is the composition of $F$ with $\frac{1}{x}: \mathbb{R} \backslash 0 \rightarrow \mathbb{R} \backslash 0$.

Lemma 2.2.11 Let $X \subset \mathbb{R}^{n}$ be a closed semi-algebraic subset. Then any continuous semi-algebraic function $F: X \rightarrow \mathbb{R}$ can be majorated 3 by the restriction to $X$ of some polynomial on $\mathbb{R}^{n}$.

Proof Let $G: \mathbb{R} \rightarrow \mathbb{R}$ be defined by $G(r):=\max _{\{x \in X|| x \mid \leq r\}} F(x)$. By Tarski-Seidenberg principle, $G$ is semi-algebraic. The lemma now reduces to its one-dimensional case, which is proven on page 43 of [BCR] (proposition 2.6.2).

\subsection{Additional preliminary results}

Theorem 2.3.1 Let $F: A \rightarrow \mathbb{R}$ be a semi-algebraic function on a locally closed semi-algebraic set. Let $Z(F):=\{x \in A \mid F(x)=0\}$ be the set of zeros of $F$ and let $A_{F}:=A \backslash Z(F)$ be its complement. Let $G: A_{F} \rightarrow \mathbb{R}$ be a semi-algebraic function.

\footnotetext{
${ }^{3}$ By majorated we mean bounded by absolute value from above.
} 
Suppose that $F$ and $G$ are continuous. Then there exists an integer $N>0$ such that the function $F^{N} G$, extended by 0 to $Z(F)$, is continuous on $A$.

The proof can be found on page 43 of [BCR] (proposition 2.6.4).

Theorem 2.3.2 (Finiteness). Let $X \subset \mathbb{R}^{n}$ be a semi-algebraic set. Then every open semi-algebraic subset of $X$ can be presented as a finite union of sets of the form $\left\{x \in X \mid p_{i}(x)>0, i=1 \ldots n\right\}$, where $p_{i}$ are polynomials in $n$ variables.

The proof can be found on page 46 of [BCR] (theorem 2.7.2).

Theorem 2.3.3 Every semi-algebraic subset of $\mathbb{R}^{n}$ has a finite number of connected components, which are semi-algebraic

The proof can be found on page 35 of [BCR] (theorem 2.4.5).

\section{Nash manifolds}

In this section we give some preliminaries on Nash manifold 4 from [BCR], [DK] and Shi] and some of their technical extensions that will be necessary for us to proceed. Most of section 4 does not rely on subsections $3.2,3.6$. However, section 5 will use all of this section.

The theory of Nash manifolds is similar both to differential topology and algebraic geometry. Our approach to Nash manifolds comes from algebraic geometry.

\subsection{Nash submanifolds of $\mathbb{R}^{n}$}

Definition 3.1.1 A Nash map from an open semi-algebraic subset $U$ of $\mathbb{R}^{n}$ to an open semi-algebraic subset $V$ of $\mathbb{R}^{m}$ is a smooth (i.e. infinitely differentiable) semialgebraic map. The ring of $\mathbb{R}$-valued Nash functions on $U$ is denoted by $\mathcal{N}(U)$. A Nash diffeomorphism is a Nash bijective map whose inverse map is also Nash.

Remark 3.1.1 In fact, a Nash map is always real analytic (cf. [Mall] or [Shi], Corollary I.5.7) but we will not use this.

Remark 3.1.2 A Nash map which is a diffeomorphism is a Nash diffeomorphism, since the inverse of a semi-algebraic map is semi-algebraic. Note also that a partial derivative of Nash function is Nash by the Tarski-Seidenberg principle.

As we are going to work with semi-algebraic differential geometry, we will need a semi-algebraic version of implicit function theorem.

\footnotetext{
${ }^{4}$ What we mean by Nash manifold is sometimes called $C^{\infty}$ Nash manifold or $C^{\omega}$ Nash manifold.
} 
Theorem 3.1.3 (Implicit Function Theorem) Let $\left(x^{0}, y^{0}\right) \in \mathbb{R}^{n+p}$ and let $f_{1}, \ldots, f_{p}$ be Nash functions on an open neighborhood of $\left(x^{0}, y^{0}\right)$ such that $f_{j}\left(x^{0}, y^{0}\right)=0$ for $j=1, . ., p$ and the matrix $\left[\frac{\partial f_{j}}{\partial y_{i}}\left(x^{0}, y^{0}\right)\right]$ is invertible. Then there exist open semialgebraic neighborhoods $U$ and $V$ of $x^{0}$ (resp. $y^{0}$ ) in $\mathbb{R}^{n}$ (resp. $\mathbb{R}^{p}$ ) and a Nash mapping $\nu$, such that $\nu\left(x^{0}\right)=y^{0}$ and $f_{1}(x, y)=\cdots=f_{p}(x, y)=0 \Leftrightarrow y=\nu(x)$ for every $(x, y) \in U \times V$.

The proof can be found on page 57 of [BCR] (corollary 2.9.8).

Definition 3.1.2 A Nash submanifold of $\mathbb{R}^{\mathbf{n}}$ is a semi-algebraic subset of $\mathbb{R}^{n}$ which is a smooth submanifold.

By the implicit function theorem it is easy to see that this definition is equivalent to the following one, given in $[\mathrm{BCR}]$ :

Definition 3.1.3 A semi-algebraic subset $M$ of $\mathbb{R}^{n}$ is said to be a Nash submanifold of $\mathbb{R}^{\mathbf{n}}$ of dimension $d$ if, for every point $m$ of $M$, there exists a Nash diffeomorphism $\nu$ from an open semi-algebraic neighborhood $\Omega$ of the origin in $\mathbb{R}^{n}$ onto an open semi-algebraic neighborhood $\Omega^{\prime}$ of $m$ in $\mathbb{R}^{n}$ such that $\nu(0)=m$ and $\left.\nu\left(\mathbb{R}^{d} \times\{0\}\right) \cap \Omega\right)=M \cap \Omega^{\prime}$.

Theorem 3.1.4 5 Any Nash submanifold of $\mathbb{R}^{n}$ is Nash diffeomorphic to a closed Nash submanifold of $\mathbb{R}^{N}$.

For proof see Corollary I.4.3 in [Shi] or theorems 8.4.6 and 2.4.5 in [BCR].

Definition 3.1.4 A Nash function on a Nash submanifold $M$ of $\mathbb{R}^{n}$ is a semialgebraic smooth function on $M$. The ring of $\mathbb{R}$-valued Nash functions on $M$ is denoted by $\mathcal{N}(M)$.

The rest of section 3 is not necessary for readers interested only in Schwartz functions on affine Nash manifolds.

\subsection{Restricted topological spaces and sheaf theory over them.}

Now we would like to define Nash manifolds independently of their embedding into $\mathbb{R}^{n}$. Analogously to algebraic geometry we will define them as ringed spaces. Hence we will need to introduce topology and structure sheaf on Nash manifolds. The natural topology to consider is topology of open semi-algebraic sets. Unfortunately, infinite unions of semi-algebraic sets are not necessary semi-algebraic, hence it is

\footnotetext{
${ }^{5}$ We will use this theorem since it makes several formulations and proofs shorter, but in each case it can be avoided.
} 
not a topology in the usual sense of the word. Therefore, we will need to define a different notion of topology and introduce an appropriate sheaf theory over it. In this section we follow [DK]. A similar use of restricted topology appears in [Mor] and $[$ Pre $]$.

Definition 3.2.1 A restricted topological space $M$ is a set $M$ equipped with a family $\stackrel{\circ}{\mathfrak{S}}(M)$ of subsets of $M$, called the open subsets which contains $M$ and the empty set, and is closed with respect to finite unions and finite intersections.

Remark 3.2.1 Pay attention that in general, there is no closure in restricted topology since infinite intersection of closed sets does not have to be closed. In our case, open sets will have closure.

Remark 3.2.2 A restricted topological space $M$ can be considered as a site in the sense of Grothendieck. The category of the site has the open sets of $M$ as objects and the inclusions as morphisms. The covers $\left(U_{i} \rightarrow U\right)_{i \in I}$ are the finite systems of inclusions with $\bigcup_{i=1}^{n} U_{i}=U$. The standard machinery of Grothendieck topology gives us the notions of a pre-sheaf and a sheaf on $M$. Now we will repeat the definitions of these notions in simpler terms.

Definition 3.2.2 A pre-sheaf $F$ on a restricted topological space $M$ is an assignment $U \mapsto F(U)$ for every open $U$ of an abelian group, vector space, etc., and for every inclusion of open sets $V \subset U$ a restriction morphism res reV $_{U}: F(U) \rightarrow F(V)$ such that res $_{U, U}=I d$ and for $W \subset V \subset U$, res $s_{V, W} \circ \operatorname{res}_{U, V}=r e s_{U, W}$.

Definition 3.2.3 A sheaf $F$ on a restricted topological space $M$ is a pre-sheaf fulfilling the usual sheaf conditions, except that now only finite open covers are admitted. The conditions are: for any open set $U$ and any finite cover $U_{i}$ of $M$ by open subsets, the sequence

$$
0 \rightarrow F(U) \stackrel{r e s_{1}}{\rightarrow} \prod_{i=1}^{n} F\left(U_{i}\right) \stackrel{r e s_{2}}{\rightarrow} \prod_{i=1}^{n-1} \prod_{j=i+1}^{n} F\left(U_{i} \cap U_{j}\right)
$$

is exact.

The map res $s_{1}$ above is defined by $r e s_{1}(\xi)=\prod_{i=1}^{n} \operatorname{res}_{U, U_{i}}(\xi)$ and the map res $s_{2}$ by

$$
\operatorname{res}_{2}\left(\prod_{i=1}^{n} \xi_{i}\right)=\prod_{i=1}^{n-1} \prod_{j=i+1}^{n} \operatorname{res}_{U_{i}, U_{i} \cap U_{j}}\left(\xi_{i}\right)-\operatorname{res}_{U_{j}, U_{i} \cap U_{j}}\left(\xi_{j}\right)
$$


Definition 3.2.4 An $\mathbb{R}$-space is a pair $\left(M, \mathcal{O}_{M}\right)$ where $M$ is a restricted topological space and $\mathcal{O}_{M}$ a sheaf of $\mathbb{R}$-algebras over $M$ which is a subsheaf of the sheaf $C_{M}$ of all continuous real-valued functions on $M$.

A morphism between $\mathbb{R}$-spaces $\left(M, \mathcal{O}_{M}\right)$ and $\left(N, \mathcal{O}_{N}\right)$ is a continuous map $\nu: M \rightarrow N$, such that for every open set $U \subset N$ and every function $f \in \mathcal{O}_{N}(U)$, the composition $\left.f \circ \nu\right|_{\nu^{-1}(U)}$ lies in $\mathcal{O}_{M}\left(\nu^{-1}(U)\right)$.

Remark 3.2.3 In a dual way, one can define the notion of a cosheaf over a restricted topological space. We will use this notion only in section 5 and give the exact definition in subsection A.4.

\subsection{Abstract Nash manifolds}

Example 3.3.1 To any Nash submanifold $M$ of $\mathbb{R}^{n}$ we associate an $\mathbb{R}$-space in the following way. Take for $\stackrel{\mathfrak{S}}{\mathfrak{S}}(M)$ the family of all open subsets of $M$ which are semialgebraic in $\mathbb{R}^{n}$. For any open (semi-algebraic) subset $U$ of $M$ we set $\mathcal{O}_{M}(U)$ to be the algebra $\mathcal{N}(U)$ of Nash functions $U \rightarrow \mathbb{R}$.

Definition 3.3.1 An affine Nash manifold is an $\mathbb{R}$-space which is isomorphic to an $\mathbb{R}$-space associated to a closed Nash submanifold of $\mathbb{R}^{n}$.

Definition 3.3.2 A Nash manifold is an $\mathbb{R}$-space $\left(M, \mathcal{N}_{M}\right)$ which has a finite cover $\left(M_{i}\right)_{i=1}^{n}$ by open sets $M_{i}$ such that the $\mathbb{R}$-spaces $\left(M_{i},\left.\mathcal{N}_{M}\right|_{M_{i}}\right)$ are affine Nash manifolds. A morphism between Nash manifolds is a morphism of $\mathbb{R}$-spaces between them.

Remark 3.3.2 A map between two closed Nash submanifolds of $\mathbb{R}^{n}$ is Nash if and only if it is a morphism of Nash manifolds. Hence we will call morphisms of Nash manifolds Nash maps, and isomorphisms of Nash manifolds Nash diffeomorphisms.

The following proposition is a direct corollary of theorem 3.1 .4 .

Proposition 3.3.3 1. Any open (semi-algebraic) subset $U$ of an affine Nash manifold $M$ is an affine Nash manifold.

2. Any open (semi-algebraic) subset $U$ of a Nash manifold $M$ is a Nash manifold.

\subsubsection{Examples and Remarks}

Example 3.3.4 Any smooth affine algebraic variety over $\mathbb{R}$ is an affine Nash manifold. 
Remark 3.3.5 A union of several connected components of an affine Nash manifold is an affine Nash manifold. This is true since every semi-algebraic subset of $\mathbb{R}^{n}$ has a finite number of connected components and each of them is semi-algebraic (see $[B C R]$, theorem 2.4.5).

Remark 3.3.6 Any affine Nash manifold is Nash diffeomorphic to a smooth real affine algebraic variety (see Chapter 14 of [BCR] for compact Nash manifold, and remark VI.2.11 in [Shi] for non-compact Nash manifold). However, the category of affine Nash manifolds is richer than the category of smooth real affine algebraic varieties, because it has more morphisms. In particular, two non-isomorphic smooth real affine algebraic varieties can be Nash diffeomorphic. For example, the hyperbola $\{x y=1\}$ is Nash diffeomorphic to the union of two straight lines $\{x+y=1 / 2\} \bigsqcup\{x+$ $y=-1 / 2\}$.

Remark 3.3.7 Note that the Nash groups $\left(\mathbb{R}_{>0}, \times\right)$ and $(\mathbb{R},+)$ are not isomorphic as Nash groups, although they are both Nash diffeomorphic and isomorphic as Lie groups.

Remark 3.3.8 Any quasiprojective Nash manifold is affine since any projective Nash manifold is affine (see page 23 in [Shi] after the proof of lemma I.3.2).

Remark 3.3.9 Any Nash manifold has an obvious natural structure of a smooth manifold.

\subsection{Nash vector bundles}

Definition 3.4.1 Let $\pi: M \rightarrow B$ be a Nash map of Nash manifolds. It is called a Nash locally trivial fibration with fiber $Z$ if $Z$ is a Nash manifold and there exist a finite cover $B=\bigcup_{i=1}^{n} U_{i}$ by open (semi-algebraic) sets and Nash diffeomorphisms $\nu_{i}$ of $\pi^{-1}\left(U_{i}\right)$ with $U_{i} \times Z$ such that the composition $\pi \circ \nu_{i}^{-1}$ is the natural projection.

Definition 3.4.2 Let $M$ be a Nash manifold. As in differential geometry, a Nash vector bundle $E$ over $M$ is a Nash locally trivial fibration with linear fiber and such that trivialization maps $\nu_{i}$ are fiberwise linear. By abuse of notation, we use the same letters to denote bundles and their total spaces.

Definition 3.4.3 Let $M$ be a Nash manifold and $E$ a Nash bundle over $M$. A Nash section of $E$ is a section of $E$ which is a Nash map.

Remark 3.4.1 In some books, for example [BCR] such a bundle is called pre-Nash. In these books, a bundle is called Nash if it can be embedded into a trivial one. 
For affine manifold $M$ this property implies that for open $U \subset M$ there exists a finite open cover $U=\cup U_{i}$ such that for any $i$, any Nash section $s: U_{i} \rightarrow E$ is a combination $s=\left.\sum f_{j} t_{j}\right|_{U_{i}}$ where $t_{j}: M \rightarrow E$ are global Nash sections and $f_{j} \in \mathcal{N}\left(U_{i}\right)$ are Nash functions on $U_{i}$. For proof see $[B C R]$.

Remark 3.4.2 Direct sums, tensor products, external tensor products, the dual, exterior powers, etc., of Nash vector bundles all have canonical structures of Nash vector bundles.

Theorem 3.4.3 Tangent, normal and conormal bundles, the bundle of differential $k$-forms, etc., of a Nash manifold have canonical structures of Nash bundles.

For the construction see section A.1 in the Appendix.

Theorem 3.4.4 The bundle of densities of a Nash manifold $M$ has a canonical structure of a Nash bundle.

For the construction see subsection A.1.1 in the Appendix.

Notation 3.4.4 We denote the bundle of densities of a Nash manifold $M$ by $D_{M}$.

Notation 3.4.5 Let $E$ be a Nash bundle over $M$. We denote $\widetilde{E}:=E^{*} \otimes D_{M}$.

Definition 3.4.6 Let $M$ be a Nash manifold. Then a Nash vector field on $M$ is a Nash section of the tangent bundle of $M$. A Nash covector field on $M$ is a Nash section of the cotangent bundle of $M$. A Nash differential $k$-form on $M$ is a Nash section of the bundle of differential $k$-forms on $M$.

Proposition 3.4.5 Let $M \subset \mathbb{R}^{n}$ be a closed Nash submanifold. Then the space of Nash covector fields on $M$ is generated over $\mathcal{N}(M)$ by $d x_{i}$.

For the proof see proposition A.1.3 in the Appendix.

\subsection{Nash differential operators}

As we have seen in the introduction, classical Schwartz functions can be defined using polynomial differential operators. In order to define Schwartz functions on Nash manifolds, we will use Nash differential operators, that will be defined in this subsection.

Definition 3.5.1 Let $M$ be a smooth real affine algebraic variety. The algebra of algebraic differential operators on $M$ is the subalgebra with 1 of $H_{\text {om }}\left(C^{\infty}(M), C^{\infty}(M)\right)$ generated by multiplications by polynomial functions and by derivations along algebraic vector fields. 
Let $M$ be an affine Nash manifold. The algebra of Nash differential operators on $M$ is the subalgebra with 1 of $\operatorname{Hom}_{\mathbb{R}}\left(C^{\infty}(M), C^{\infty}(M)\right)$ generated by multiplications by Nash functions and by derivations along Nash vector fields.

The following lemma immediately follows from the Tarski-Seidenberg theorem.

Lemma 3.5.1 Any Nash differential operator maps $\mathcal{N}(M)$ to $\mathcal{N}(M)$.

Remark 3.5.2 One could give equivalent definition of the algebra of Nash differential operators on $M$ as Grothendieck's algebra of differential operators over the algebra $\mathcal{N}(M)$.

We will use the following trivial lemma

Lemma 3.5.3 Let $U \subset \mathbb{R}^{n}$ be open (semi-algebraic) subset. Then any Nash differential operator $D$ on $U$ can be written as $\sum_{i=1}^{k} f_{i}\left(\left.D_{i}\right|_{U}\right)$ where $f_{i}$ are Nash functions on $U$ and $D_{i}$ are Nash differential operators on $\mathbb{R}^{n}$.

Remark 3.5.4 A similar but weaker lemma holds for any affine Nash manifold and its open subset. It can be proven using remark 3.4.1.

\subsubsection{Algebraic differential operators on a Nash manifold}

The following ad hoc definition will be convenient for us for technical reasons.

Definition 3.5.2 Given a closed embedding of $M$ to $\mathbb{R}^{n}$ we can define the notion of algebraic differential operators on $M$, which depends on the embedding. Algebraic vector fields on $M$ are defined as Nash vector fields obtained by composition of the restriction to $M$ and the orthogonal projection to $T_{M}$ from algebraic vector fields on $\mathbb{R}^{n}$. The algebra of algebraic differential operators on $M$ is the subalgebra with 1 of $\mathrm{Hom}_{\mathbb{R}}\left(C^{\infty}(M), C^{\infty}(M)\right)$ generated by multiplications by restriction of polynomial functions and by derivations along algebraic vector fields.

Lemma 3.5.5 Let $M \subset \mathbb{R}^{n}$ be a closed affine Nash submanifold. Then the space of Nash differential operators on $M$ is generated as a module over $\mathcal{N}(M)$ by algebraic differential operators.

Proof. Consider the algebraic vector fields $\left.\frac{\partial}{\partial x_{i}}\right|_{M}$ obtained from the standard vector fields $\frac{\partial}{\partial x_{i}}$ on $\mathbb{R}^{n}$ by restriction to $M$ and orthogonal projection to $T_{M}$. By proposition

3.4.5, $\left.\frac{\partial}{\partial x_{i}}\right|_{M}$ generate Nash vector fields over $\mathcal{N}(M)$. The lemma now follows from lemma 3.5.1, the chain rule and the Leibnitz rule. 


\subsection{Nash tubular neighborhood}

We will need a Nash analog of the tubular neighborhood theorem from differential geometry.

Notation 3.6.1 Let $M$ be a Nash manifold. Let $Z \subset M$ be a closed Nash submanifold. We denote by $N_{Z}^{M}$ the normal bundle of $Z$ in $M$. In case $M$ is equipped with a Nash Riemannian metric, we consider $N_{Z}^{M}$ as a subbundle of the tangent bundle of $M$.

Notation 3.6.2 Let $x \in \mathbb{R}^{n}$ and $r \in \mathbb{R}$. We denote the open ball with center $x$ and radius $r$ by $B(x, r)$.

Let $M$ be a Nash manifold with a Nash Riemannian metric on it. Let $Z \subset$ $M$ be a closed Nash submanifold and $F: Z \rightarrow \mathbb{R}$ a function on it. We define $B_{M}(Z, F) \subset N_{Z}^{M}$ by $B_{M}(Z, F):=\left\{(z, v) \in N_{Z}^{M} \mid\|v\|<F(z)\right\}$.

Remark 3.6.1 Note that if $F$ is strictly positive and semi-algebraic then $B_{M}(Z, F)$ is Nash.

Definition 3.6.3 Let $M$ be a Nash manifold with a Nash Riemannian metric on it. Let $Z \subset M$ be a closed Nash submanifold. A Nash tubular neighborhood of $Z$ in $M$ is the following data: an open Nash neighborhood Tube $(Z, M)$ of $Z$ in $M$, a strictly positive Nash function $\rho_{Z}^{M} \in \mathcal{N}(Z)$ and a Nash diffeomorphism $\nu_{Z}^{M}: B_{M}\left(Z, \rho_{Z}^{M}\right) \simeq \operatorname{Tube}(Z, M)$.

Theorem 3.6.2 (Nash Tubular Neighborhood). Let $Z \subset M \subset \mathbb{R}^{n}$ be closed affine Nash submanifolds. Equip $M$ with the Riemannian metric induced from $\mathbb{R}^{n}$. Then $Z$ has a Nash tubular neighborhood. Moreover, for any closed Nash submanifold $Y \subset M$ disjoint from $Z, Z$ has a Nash tubular neighborhood in $M \backslash Y$.

Proof. Denote by $\nu_{1}$ the map $\nu_{1}: N_{Z}^{M} \rightarrow \mathbb{R}^{n}$ given by $\nu_{1}(z, v)=z+v$. Denote by $W$ the set of all points $x \in \mathbb{R}^{n}$ such that there exists a unique point of $M$ closest to $x$. Denote $U:=\stackrel{o}{W}$. It is open semi-algebraic, and the projection $p r: U \rightarrow M$ is also semi-algebraic. Denote $V=\nu_{1}^{-1}(U)$ and $\nu=p r \circ \nu_{1}: V \rightarrow M$.

From differential topology we know that there exists a smooth strictly positive function $\sigma$ such that $\left.\nu\right|_{B_{M}(Z, \sigma)}$ is a diffeomorphism to its image. For any $z \in Z$ denote $Z_{z}:=B(z, 1) \cap Z$. For any $z \in Z$ there exists $\varepsilon>0$ such that $\left.\nu\right|_{B_{M}\left(Z_{z}, \varepsilon\right)}$ is a diffeomorphism to its image, for example $\varepsilon=\min _{Z_{z}}(\sigma)$. Denote by $G(z)$ the supremum of all such $\varepsilon$. Let $\rho_{Z}^{M}(z):=\min (G(z) / 2,1 / 10), \operatorname{Tube}(Z, M):=\nu\left(B\left(Z, \rho_{Z}^{M}\right)\right)$ and $\nu_{Z}^{M}:=\left.\nu\right|_{B\left(Z, \rho_{Z}^{M}\right)}$. It is easy to see that $\left(T u b e(Z, M), \rho_{Z}^{M}, \nu_{Z}^{M}\right)$ form a Nash tubular neighborhood.

Clearly we can always find $\rho_{Z}^{M \backslash Y}<\rho_{Z}^{M}$ such that the corresponding Nash tubular neighborhood in $M$ does not intersect $Y$. 
Corollary 3.6.3 Let $M$ be an affine Nash manifold and $Z \subset M$ be closed affine Nash submanifold. Then $Z$ has a neighborhood $U$ in $M$ which is Nash diffeomorphic to the total space of the normal bundle $N_{Z}^{M}$.

This corollary follows from the theorem using the following technical lemma.

Lemma 3.6.4 Let $E \rightarrow M$ be a Nash bundle. Let $f \in \mathcal{N}(E)$ be a Nash fiberwise homogeneous function of even degree $l \geq 2$. Suppose $f(M)=\{0\}$ and $f(E \backslash M)>0$. Let $U:=\{x \in E \mid f(x)<1\}$. Then $U$ is Nash diffeomorphic to $E$.

Proof. Define a Nash diffeomorphism by stereographic projection, i.e. $\nu: U \rightarrow E$ by $\nu(m, v)=\left(m, \frac{1}{1-f(m, v)} v\right)$.

\section{Schwartz and tempered functions on affine Nash manifolds}

\subsection{Schwartz functions}

Definition 4.1.1 Let $M$ be an affine Nash manifold. We define the space of Schwartz functions on $M$ by $\mathcal{S}(M):=\left\{\phi \in C^{\infty}(M) \mid\right.$ for any Nash differential operator $D$ on $M, D \phi$ is bounded $\}$. We introduce a topology on this space by the following system of semi-norms : $\|\phi\|_{D}:=\sup _{x \in M}|D \phi(x)|$.

Proposition 4.1.1 Let $M \subset \mathbb{R}^{n}$ be a closed affine Nash submanifold, and $\phi$ be a smooth function on $M$. Suppose that for any algebraic differential operator $D$ on $M, D \phi$ is bounded. Then $\phi \in \mathcal{S}(M)$.

Proof. Let $D^{\prime}$ be a Nash differential operator. By lemma 3.5.5, $D^{\prime} \phi=\sum_{i=1}^{n} g_{i} D_{i} \phi$ where $D_{i}$ are algebraic differential operators on $M$ and $g_{i}$ are Nash functions on $M$. By lemma 2.2.11, $g_{i}$ can be majorated by polynomials $h_{i}$, hence $\left|D^{\prime} \phi\right|=\left|\sum g_{i} D_{i} \phi\right| \leq$ $\sum_{i=1}^{n}\left|\left(h_{i} D_{i}\right) \phi\right|$. The functions $\left(h_{i} D_{i}\right) \phi$ are bounded since $h_{i} D_{i}$ is also an algebraic differential operator.

Corollary 4.1.2 $\mathcal{S}(M)$ is a Fréchet space. 6

Corollary 4.1.3 If $M$ is a smooth algebraic variety then $\mathcal{S}(M)$ can be defined as the space of smooth functions $\phi$ such that $D \phi$ is bounded for every algebraic differential operator D on $M$. In particular, $\mathcal{S}\left(\mathbb{R}^{n}\right)$ is the space of classical Schwartz functions.

\footnotetext{
${ }^{6}$ This follows from the proof of the previous proposition rather than from its formulation.
} 
Remark 4.1.4 It is easy to see that:

1. Nash differential operators act on Schwartz functions.

2. Schwartz functions form an algebra.

Remark 4.1.5 One could give an equivalent definition of Schwartz functions using a system of $L^{2}$ norms. Namely, choosing a Nash non-vanishing density $\mu$ and defining $\|\phi\|_{D, \mu}:=\int_{M}|D \phi|^{2} d \mu$.

\subsection{Tempered functions}

Definition 4.2.1 Let $M$ be an affine Nash manifold. A function $\alpha \in C^{\infty}(M)$ is said to be tempered if for any Nash differential operator $D$ there exists a Nash function $f$ such that $|D \alpha| \leq f$.

Proposition 4.2.1 Let $M$ be an affine Nash manifold and $\alpha$ be a tempered function on $M$. Then $\alpha \mathcal{S}(M) \subset \mathcal{S}(M)$.

Proof. Let $\phi$ be a Schwartz function and $D$ be a Nash differential operator on $M$. By the Leibnitz rule, $D(\alpha \phi)=\sum_{i=1}^{n}\left(D_{i} \alpha\right)\left(D_{i}^{\prime} \phi\right)$ for some Nash differential operators $D_{i}$ and $D_{i}^{\prime}$. Since $\alpha$ is tempered, there exist positive Nash functions $f_{i}$ such that $\left|D_{i} \alpha\right| \leq f_{i}$. Denote $D_{i}^{\prime \prime}:=f_{i} D_{i}^{\prime}$. So $|D(\alpha \phi)|=\left|\sum_{i=1}^{n}\left(D_{i} \alpha\right)\left(D_{i}^{\prime} \phi\right)\right| \leq \sum_{i=1}^{n}\left|f_{i} D_{i}^{\prime} \phi\right|=$ $\sum_{i=1}^{n}\left|D_{i}^{\prime \prime} \phi\right|$, which is bounded since $\phi$ is Schwartz.

Remark 4.2.2 One can prove that the converse statement is also true, namely if $\alpha \mathcal{S}(M) \subset \mathcal{S}(M)$ then $\alpha$ is tempered. We will neither use nor prove that in this paper.

The proof of the following lemma is straightforward.

Lemma 4.2.3 1. Nash differential operators act on tempered functions.

2. Tempered functions form a unitary algebra and every tempered function whose absolute value is bounded from below by a strictly positive constant is invertible in this algebra.

\subsection{Extension by zero of Schwartz functions}

Proposition 4.3.1 (Extension by zero). Let $M$ be an affine Nash manifold and $U \subset M$ be an open (semi-algebraic) subset. Then the extension by zero of a Schwartz function on $U$ is a Schwartz function on $M$ which vanishes with all its derivatives outside $U$. 
The proposition follows by induction from the following lemma.

Lemma 4.3.2 For any $\phi: U \rightarrow \mathbb{R}$ denote by $\widetilde{\phi}: M \rightarrow \mathbb{R}$ its extension by 0 outside $U$. Let $\phi \in \mathcal{S}(U)$. Then $\widetilde{\phi}$ is differentiable at least once and for any Nash differential operator $D$ of order 1 on $M, D \widetilde{\phi}=\widetilde{\left.D\right|_{U} \phi}$.

Proof. We have to show that for any $z \in M \backslash U, \widetilde{\phi}$ is differentiable at least once at $z$ and its derivative at $z$ in any direction is 0 . Embed $M \hookrightarrow \mathbb{R}^{n}$ and denote $F_{z}(x):=\|x-z\|$. Clearly, $1 / F_{z}^{2} \in \mathcal{N}(U)$. Hence $\phi / F_{z}^{2}$ is bounded in $U$ and therefore $\widetilde{\phi} / F_{z}^{2}$ is bounded on $M \backslash z$, which finishes the proof.

\subsection{Partition of unity}

In the proofs in this subsection we use the technical cover tools developed in appendix (subsection A.3).

Theorem 4.4.1 (Partition of unity). Let $M$ be an affine Nash manifold, and let $\left(U_{i}\right)_{i=1}^{n}$ be a finite open (semi-algebraic) cover of $M$. Then

1) there exist tempered functions $\alpha_{1}, \ldots, \alpha_{n}$ on $M$ such that $\operatorname{supp}\left(\alpha_{i}\right) \subset U_{i}, \sum_{i=1}^{n} \alpha_{i}=$ 1.

2) Moreover, we can choose $\alpha_{i}$ in such a way that for any $\phi \in \mathcal{S}(M), \alpha_{i} \phi \in \mathcal{S}\left(U_{i}\right)$.

Proof of 1. By lemma A.3.1, which follows from the finiteness theorem, we can assume $U_{i}=\left\{x \in M \mid F_{i}(x) \neq 0\right\}$ and $\left.F_{i}\right|_{U_{i}}$ is a positive Nash function. By proposition A.3.2, there exists a strictly positive Nash function $f$ such that the sets $V_{i}=\left\{x \in M \mid F_{i}(x)>f(x)\right\}$ also cover $M$. Let $\rho: \mathbb{R} \rightarrow[0,1]$ be a smooth function such that $\rho((-\infty, 0.1])=\{0\}, \rho([1, \infty))=\{1\}$. Define $\beta_{i}:=\rho\left(F_{i} / f\right)$. It is easy to see that they are tempered on $M$. Denote $\alpha_{i}:=\frac{\beta_{i}}{\sum \beta_{i}}$. The functions $\beta_{i}$ are tempered, $\sum \beta_{i} \geq 1$ hence $\alpha_{i}$ are tempered functions by lemma 4.2.3. Hence the $\alpha_{i}$ form a tempered partition of unity.

In the proof of part 2 we will need the following technical lemma.

Lemma 4.4.2 Let $M \subset \mathbb{R}^{d}$ be an affine closed Nash submanifold and

$$
U=\left\{x \in M \mid \forall 1 \leq i \leq n, p_{i}(x)>0\right\}
$$

where $p_{i}$ are polynomials on $\mathbb{R}^{d}$. Let $g>0$ be a Nash function and

$$
U^{\prime}=\left\{x \in M \mid \forall 1 \leq i \leq n, p_{i}(x)>g(x)\right\} .
$$

Then any Schwartz function $\phi$ on $M$ which is 0 outside $U^{\prime}$ is a Schwartz function on $U$. 
Proof. There is a standard closed embedding $\nu^{\prime}: U \hookrightarrow \mathbb{R}^{d+n}$ whose last $n$ coordinates are defined by $1 / p_{i}$. By proposition 4.1.1, it is enough to check that for any differential operator $D$ on $U$ algebraic with respect to $\nu^{\prime},\left.D \phi\right|_{U}$ is bounded. Standard algebraic geometry arguments show that $D$ is a sum of differential operators of the form $\frac{1}{\prod p_{i}^{k}}\left(\left.D^{\prime}\right|_{U}\right)$ where $k$ is a natural number and $D^{\prime}$ is a differential operator on $M$ algebraic with respect to the imbedding $M \subset \mathbb{R}^{d}$. The lemma follows now from the fact that inside $U^{\prime}, \frac{1}{\prod p_{i}} \leq g^{n}$.

Remark 4.4.3 In fact, more is true: any Schwartz function on $M$ which vanishes outside $U$ together with all its derivatives is Schwartz on $U$. We will prove that in subsection 5.4, but the proof uses partition of unity.

Proof of part 2 of theorem 4.4.1. Fix a closed imbedding $M \subset \mathbb{R}^{d}$. By finiteness theorem (2.3.2), we can suppose $U_{i}=\left\{x \in M \mid p_{i}(x)>0\right\}$ where $p_{i}$ are polynomials on $\mathbb{R}^{d}$. By proposition A.3.2, there exists a Nash function $g>0$ such that the sets $V_{i}=\left\{x \in M \mid p_{i}(x)>g\right\}$ also cover $M$. Let $\alpha_{i}$ be the tempered partition of unity for the cover $V_{i}$. By the previous lemma, $\alpha_{i}$ satisfy 2 .

Definition 4.4.1 Let $M$ be an affine Nash manifold. We define the cosheaf $\mathcal{S}_{M}$ of Schwartz functions on $M$ in the following way. For any open (semi-algebraic) subset $U$ define $\mathcal{S}_{M}(U)$ to be $\mathcal{S}(U)$ and for $V \subset U$ define the extension map ext ${ }_{V, U}$ : $\mathcal{S}_{M}(V) \rightarrow \mathcal{S}_{M}(U)$ to be the extension by zero.

Proposition 4.4.4 $\mathcal{S}_{M}$ is a cosheaf (in the restricted topology).

Proof. It follows from partition of unity (theorem 4.4.1) and extension by zero (proposition 4.3.1).

\subsection{Restriction and sheaf property of tempered functions}

In the proofs given in this subsection we use the technical tools developed in the Appendix (subsection A.2).

Theorem 4.5.1 (Tempered restriction). Let $M \subset \mathbb{R}^{n}$ be an affine Nash manifold, $p_{1}, \ldots, p_{n}$ be polynomials on $\mathbb{R}^{n}$ and $U=U_{p_{1}, \ldots, p_{n}}=\left\{x \in M \mid p_{1}(x)>0, \ldots, p_{n}>0\right\}$ be a basic open semi-algebraic subset. Then the restriction to $U$ of any tempered function $\alpha$ on $M$ is tempered.

Proof. It is enough to prove the statement for $n=1$. Denote $p=p_{1}$. Let $D$ be a Nash differential operator on $U$. We will prove that $|D \alpha|$ is bounded by some Nash function.

Step 1. Reduction to the case $D=\left.f D^{\prime}\right|_{U}$, where $D^{\prime}$ is a Nash differential operator 
on $M$ and $f$ is a Nash function on $U$.

By lemma 3.5.5, we may assume that there exist an algebraic differential operator $D_{1}$ and a Nash function $f_{1}$ on $U$ such that $D=f_{1} D_{1}$. From algebraic geometry we know that there exist a natural number $n$ and an algebraic differential operator $D^{\prime}$ on $M$ such that $D_{1}=\left.p^{-n} D^{\prime}\right|_{U}$. Now we take $f=f_{1} p^{-n}$.

Step 2. Proof of the theorem.

Since $\alpha$ is a tempered function on $M$, there exists a positive Nash function $f_{2}$ on $M$ such that $\left|D^{\prime} \alpha\right| \leq f_{2}$. So $|D \alpha| \leq\left.|f| \cdot f_{2}\right|_{U}$. By lemma A.2.1 there exists a Nash function $f_{3}$ on $U$ such that $\left.|f| \cdot f_{2}\right|_{U} \leq f_{3}$ and hence also $|D \alpha| \leq f_{3}$.

Theorem 4.5.2 (The sheaf property of tempered functions). Let $M$ be an affine Nash manifold and $\left(U_{i}\right)_{i=1}^{n}$ its open cover. Let $\alpha$ be a smooth function on $M$. Suppose $\left.\alpha\right|_{U_{i}}$ is tempered on $U_{i}$. Then $\alpha$ is tempered on $M$.

Proof. Embed $M \hookrightarrow \mathbb{R}^{n}$ and let $d$ be the global metric induced from $\mathbb{R}^{n}$. Let $D$ be a Nash differential operator on $M$. We know that there exist strictly positive Nash functions $f_{i}$ on $U_{i}$ such that $|D \alpha| \leq f_{i}$ on $U_{i}$. Let $G_{i}(x):=\min \left(f_{i}(x)^{-1}, d\left(x, M \backslash U_{i}\right)\right)$. Extend $G_{i}$ by zero to $M$ and define $G:=\max G_{i}$. Let $F:=G^{-1}$ and let $f$ be a Nash function that majorates $F$, which exists by lemma A.2.1. It is easy to see that $|D \alpha| \leq f$.

Definition 4.5.1 Let $M$ be an affine Nash manifold. We define the sheaf $\mathcal{T}_{M}$ of tempered functions on $M$ in the following way. For any open (semi-algebraic) subset $U$ define $\mathcal{T}_{M}(U)$ to be $\mathcal{T}(U)$ and for $V \subset U$ define the usual restriction map $\operatorname{res}_{U, V}: \mathcal{T}_{M}(V) \rightarrow \mathcal{T}_{M}(U)$.

We can summarize the previous 2 theorems in the following proposition:

Proposition 4.5.3 $\mathcal{T}_{M}$ is a sheaf (in the restricted topology).

\subsection{Extension of Schwartz and tempered functions from closed submanifolds}

Theorem 4.6.1 Let $M$ be an affine Nash manifold and $Z \hookrightarrow M$ be a closed Nash submanifold. The restriction $\mathcal{S}(M) \rightarrow \mathcal{S}(Z)$ is defined, continuous and onto. Moreover, it has a section $s: \mathcal{S}(Z) \rightarrow \mathcal{S}(M)$ such that if $\phi \in \mathcal{S}(Z)$ is 0 at some point $p$ with all its derivatives, then $s(\phi)$ is also 0 at $p$ with all its derivatives.

Proof. Clearly, the restriction is well defined and continuous. Let us show that it is onto. 
Case $1 M=N \times \mathbb{R}^{n}, Z=N \times\{0\}$.

Choose a Schwartz function $\psi \in \mathcal{S}\left(\mathbb{R}^{n}\right)$ such that $\psi=1$ in a neighborhood of the origin. For any $\phi \in \mathcal{S}(Z)$ we define $s(\phi)(n, v):=\phi(n, 0) \psi(v)$. Clearly, $s$ is the required section.

Case 2 There exists a Nash diffeomorphism between $M$ and a Nash vector bundle over $Z$ which maps $Z$ to the zero section.

In this the claim follows from case 1 and theorem 4.4.1.

Case 3 General.

Follows from case 2, corollary 3.6 .3 of the Nash tubular neighborhood theorem and extension by zero 4.3.1.

In the same way one can prove an analogous theorem for tempered functions.

Theorem 4.6.2 Let $Z \hookrightarrow M$ be a closed Nash embedding of affine Nash manifolds. The restriction $\mathcal{T}(M) \rightarrow \mathcal{T}(Z)$ is defined, continuous and onto. Moreover, it has a section $s: \mathcal{T}(Z) \rightarrow \mathcal{T}(M)$ such that if $\alpha \in \mathcal{T}(Z)$ is 0 at some point $p$ with all its derivatives, then $s(\alpha)$ is also 0 at $p$ with all its derivatives.

\section{Schwartz, tempered and generalized Schwartz sections of Nash bundles over arbitrary Nash manifolds}

\subsection{Main definitions}

To define tempered and Schwartz functions on abstract Nash manifolds we use these notions on affine Nash manifolds and glue them using sheaf and cosheaf properties respectively.

Definition 5.1.1 Let $M$ be a Nash manifold, and $E$ be a Nash bundle over it. Let $M=\bigcup_{i=1}^{k} U_{i}$ be an affine Nash trivialization of $E$. A global section s of $E$ over $M$ is called tempered if for any $i$, all the coordinate components of $\left.s\right|_{U_{i}}$ are tempered functions. The space of global tempered sections of $E$ is denoted by $\mathcal{T}(M, E)$.

Proposition 5.1.1 The definition does not depend on the choice of $U_{i}$.

Proof. It follows from the sheaf properties of tempered functions (proposition 4.5.1 and theorem 4.5.2). 
Definition 5.1.2 Let $M$ be a Nash manifold, and let $E$ be a Nash bundle over it. Let $M=\bigcup_{i=1}^{k} U_{i}$ be an affine Nash trivialization of $E$. Then we have a map $\phi: \bigoplus_{i=1}^{k} \mathcal{S}\left(U_{i}\right)^{n} \rightarrow C^{\infty}(M, E)$. We define the space $\mathcal{S}(M, E)$ of global Schwartz sections of $E$ by $\mathcal{S}(M, E):=\operatorname{Im} \phi$. We define the topology on this space using the isomorphism $\mathcal{S}(M, E) \cong \bigoplus_{i=1}^{k} \mathcal{S}\left(U_{i}\right)^{n} / \operatorname{Ker} \phi$.

Proposition 5.1.2 The definition does not depend on the choice of $U_{i}$.

Proof. It follows from theorems on partition of unity (theorem 4.4.1) and extension by zero (proposition 4.3.1).

Definition 5.1.3 Let $M$ be a Nash manifold, and let $E$ be a Nash bundle over it. $W e$ define the cosheaf $\mathcal{S}_{M}^{E}$ of Schwartz sections of $E$ by $\mathcal{S}_{M}^{E}(U):=\mathcal{S}\left(U,\left.E\right|_{U}\right)$. $W e$ also define the sheaf $\mathcal{G}_{M}^{E}$ of generalized Schwartz sections of $E$ by $\mathcal{G}_{M}^{E}(U):=\left(\mathcal{S}_{M}^{\widetilde{E}}\right)^{*}$, where $\widetilde{E}=E^{*} \otimes D_{M}$, and the sheaf $\mathcal{T}_{M}^{E}$ of tempered sections of $E$ by $\mathcal{T}_{M}^{E}(U):=\mathcal{T}\left(U,\left.E\right|_{U}\right)$.

Proposition 5.1.3 $\mathcal{S}_{M}^{E}$ is a cosheaf, and $\mathcal{T}_{M}^{E}$ and $\mathcal{G}_{M}^{E}$ are sheaves.

Proof. It follows from the definitions.

Remark 5.1.4 $\mathcal{T}_{M}$ and $\mathcal{G}_{M}$ are functors from the category of Nash bundles over $M$ to the categories of sheaves on $M$. Also, $\mathcal{S}_{M}$, is a functor from the category of Nash bundles over $M$ to the categories of cosheaves on $M$. The mappings of morphisms are obvious.

\subsection{Partition of unity}

This subsection is rather similar to subsection 4.4 about partition of unity for affine Nash manifolds. In particular, in the proofs in this subsection we use the technical cover tools and definitions given in the Appendix (subsection A.3).

Theorem 5.2.1 (Partition of unity for any Nash manifold). Let $M$ be a Nash manifold, and let $\left(U_{i}\right)_{i=1}^{n}$ be a finite open cover. Then

1) there exist tempered functions $\alpha_{1}, \ldots, \alpha_{n}$ on $M$ such that $\operatorname{supp}\left(\alpha_{i}\right) \subset U_{i}, \sum_{i=1}^{n} \alpha_{i}=1$.

2) Moreover, we can choose $\alpha_{i}$ in such a way that for any $\phi \in \mathcal{S}(M), \alpha_{i} \phi \in \mathcal{S}\left(U_{i}\right)$. 


\section{Proof of 1 .}

Let $\left\{F_{j}\right\}$ be a basic collection of continuous semi-algebraic functions such that $M_{F_{j}}$ is a refinement of $\left\{U_{i}\right\}$. It exists by proposition A.3.4. Let $\rho: \mathbb{R} \rightarrow[0,1]$ be a smooth function such that $\rho((-\infty, 0.1])=\{0\}, \rho([1, \infty))=\{1\}$. Denote $\beta_{j}:=\rho \circ F_{j}$ and $\gamma_{j}=\frac{\beta_{j}}{\sum \beta_{j}}$. It is easy to see that $\gamma_{j}$ are tempered. Now for every $j$ we choose $i(j)$ such that $M_{F_{j}} \subset U_{i(j)}$. Define $\alpha_{i}:=\sum_{j \mid i(j)=i} \gamma_{j}$. It is easy to see that $\alpha_{j}$ is a tempered partition of unity.

As in the proof of affine partition of unity theorem, part 2 follows from part 1 , proposition A.3.3 and the following lemma, similar to lemma 4.4.2.

Lemma 5.2.2 Let $M$ be a Nash manifold. Let $V \subset U \subset M$ be open (semi-algebraic) sets such that $\bar{V} \subset U$. Let $\alpha$ be a tempered function on $M$ supported in $V$. Let $\phi$ be a Schwartz function on $M$. Then $\left.\alpha \phi\right|_{U}$ is a Schwartz function on $U$.

In order to prove this lemma we need the following technical lemma.

Lemma 5.2.3 Let $M$ be an affine Nash manifold. Let $\phi$ be a Schwartz function on $M$. Let $U \subset M$ be an open (semi-algebraic) subset. Denote $\phi^{\prime}:=\phi \mathbf{1}_{U}$ where $\mathbf{1}_{U}$ is the characteristic function of $U$. Suppose that $\phi^{\prime}$ is smooth. Then $\phi^{\prime}$ is Schwartz.

Proof. Denote $W:=U \cup(\bar{U})^{c}$. Let $D$ be a Nash differential operator on $M$. It is easy to see that $\left.\left|D \phi^{\prime}\right|_{W}|\leq| D \phi\right|_{W} \mid$ and $W$ is dense in $M$. Hence $\left|D \phi^{\prime}\right| \leq|D \phi|$ which is bounded.

Proof of lemma 5.2.2. Without loss of generality we can assume that $M$ is affine. Denote $W:=M \backslash \bar{V}$. Note that $M=U \cup W$. Since Schwartz functions form a cosheaf, $\phi=\phi_{U}+\phi_{W}$ where $\phi_{U}$ and $\phi_{W}$ are extensions by zero of Schwartz functions on $U$ and on $W$, respectively. Note that $\left.\phi\right|_{\bar{V}}=\left.\phi_{U}\right|_{\bar{V}}$ so $\left.\phi\right|_{U}=\left.\left.\left(\phi_{U}\right)\right|_{U} \cdot\left(\mathbf{1}_{\bar{V}}\right)\right|_{U}$ and therefore by the lemma $\left.\phi\right|_{U}$ is Schwartz.

Remark 5.2.4 Partitions of unity clearly exist for Schwartz sections, generalized Schwartz sections and tempered sections of any Nash bundle, i.e. for any Nash bundle $E$ over $M$ and any finite open cover $U_{i}$ of $M$ there exists a tempered partition of unity $\alpha_{i}$ such that for any $\phi \in \mathcal{S}(M, E), \phi \alpha_{i} \in \mathcal{S}\left(U_{i}, E\right)$ and for any $\xi \in \mathcal{G}(M, E)$, $\xi \alpha_{i} \in \mathcal{G}\left(U_{i}, E\right)$.

\subsection{Basic properties}

Let us now prove properties 1 - 5 mentioned in section 1.5 . Property 1 holds by definition. Clearly, for affine $M$ and trivial 1-dimensional $E$, $\mathcal{S}(M, E)=\mathcal{S}(M)$ and property 2 holds. Property 4 is satisfied by definition. Property 5 follows from theorems 4.6.1 and 4.6.2. 
Theorem 5.3.1 Property 3 holds, i.e. for compact Nash manifold $M, \mathcal{S}(M, E)=$ $\mathcal{T}(M, E)=C^{\infty}(M, E)$.

Proof Let $\alpha$ be a smooth section of a Nash bundle $E$ over a compact Nash manifold M. We have to show that it is also a Schwartz section. Since $M$ is a Nash manifold, any point $m \in M$ has a neighborhood $U_{m}$ Nash diffeomorphic to an open ball in $\mathbb{R}^{n}$ of radius 1. Denote by $V_{m} \subset M$ the preimage of the ball of radius 0.9 under this Nash diffeomorphism. $\left\{V_{m}\right\}_{m \in M}$ is a cover of $M$. Let us choose a finite subcover $V_{m_{i}}$. By classical partition of unity, $\alpha$ can be represented as $\alpha=\sum \alpha_{i}$ where $\operatorname{supp}\left(\alpha_{i}\right) \subset V_{m_{i}}$. $\alpha_{i}$ are clearly Schwartz on $U_{i}$. Hence $\alpha$ is Schwartz on $M$.

\subsection{Characterization of Schwartz functions on open subset}

Let us now prove property 6. First we will prove it for trivial 1-dimensional bundle.

Theorem 5.4.1 (Characterization of Schwartz functions on open subset)

Let $M$ be a Nash manifold, $Z$ be a closed (semi-algebraic) subset and $U=M \backslash Z$. Let $W_{Z}$ be the closed subspace of $\mathcal{S}(M)$ defined by $W_{Z}:=\{\phi \in \mathcal{S}(M) \mid \phi$ vanishes with all its derivatives on $Z\}$. Then restriction and extension by 0 give an isomorphism $\mathcal{S}(U) \cong W_{Z}$.

We will use the following elementary lemma from calculus.

Lemma 5.4.2 Suppose $\alpha \in C^{\infty}(\mathbb{R})$ vanishes at 0 with all its derivatives. Then for any natural number $n, \alpha(t)=t^{n} \alpha^{(n)}(\theta)$ for some $\theta \in[0, t]$.

Proof of the theorem.

Case $1 M=\mathbb{R}^{N}$.

Let $\phi \in \mathcal{S}(U)$ and let $\widetilde{\phi}$ be its extension by 0 . By proposition 4.3 .1 on extension by zero, $\widetilde{\phi} \in W$.

Now, let $\phi \in W_{Z}$. For any point $x \in \mathbb{R}^{N}$ define $r(x):=\operatorname{dist}(x, Z)$. Let $S:=S(0,1) \in \mathbb{R}^{N}$ be the unit sphere. Consider the function $\psi$ on $S \times Z \times \mathbb{R}$ defined by $\psi(s, z, t):=\phi(z+t s)$. From the previous lemma 5.4 .2 we see that $\psi(s, z, t)=\left.t^{n} \frac{\partial^{n}}{(\partial t)^{n}} \psi(x, s, t)\right|_{t=\theta}$ for some $\theta \in[0, t]$. As $\phi$ is Schwartz, it is easy to see that $\frac{\partial^{n}}{(\partial t)^{n}} \psi(x, s, t)$ is bounded on $Z \times S \times \mathbb{R}$. Therefore $|\psi(s, z, t)| \leq C|t|^{n}$ for some constant $C$ and hence $\phi / r^{n}$ is bounded on $\mathbb{R}^{N}$ for any $n$.

Let $h$ be a Nash function on $U$. By lemma 2.3.1, $r^{n} h$ extends by 0 to a continuous semi-algebraic function on $\mathbb{R}^{N}$ for $n$ big enough. It can be majorated by $f \in \mathcal{N}\left(\mathbb{R}^{N}\right)$. Therefore $|\phi h|=\left|\left(\phi / r^{n}\right) r^{n} h\right| \leq|\phi f| / r^{n}$. $\phi f \in W$, thus $|\phi f| / r^{n}$ is bounded and hence $|\phi h|$ is bounded. 
For any Nash differential operator $D$ on $\mathbb{R}^{N}, D \phi \in W$. Hence $h D \phi$ is bounded. By lemma 3.5.3, every Nash differential operator on $U$ is a sum of differential operators of the form $\left.h D\right|_{U}$, where $D$ is a Nash differential operator on $\mathbb{R}^{N}$ and $h$ a Nash function on $U$. Hence $\left.\phi\right|_{U} \in \mathcal{S}(U)$.

Case $2 M$ is affine.

Follows from the previous case and theorem 4.6.1 (extension from a closed Nash submanifold).

Case 3 General case.

Choose an affine cover of $M$. The theorem now follows from the previous case and partition of unity.

Property [6 is an immediate corollary of the previous theorem and partition of unity. Let us remind it.

Theorem 5.4.3 (Characterization of Schwartz sections on open subset)

Let $M$ be a Nash manifold, $Z$ be a closed (semi-algebraic) subset and $U=M \backslash$ $Z$. Let $W_{Z}$ be the closed subspace of $\mathcal{S}(M, E)$ defined by $W_{Z}:=\{\phi \in \mathcal{S}(M, E) \mid \phi$ vanishes with all its derivatives on $Z\}$. Then restriction and extension by 0 give an isomorphism $\mathcal{S}_{M}^{E}(U) \cong W_{Z}$.

Corollary 5.4.4 Let $E \rightarrow M$ be a Nash bundle. Then the cosheaf $\mathcal{S}_{M}^{E}$ and the sheaf $\mathcal{G}_{M}^{E}$ are flabby. In other words, let $V \subset U \subset M$ be open (semi-algebraic) subsets. Then ext ${ }_{V, U}: \mathcal{S}_{M}^{E}(V) \rightarrow \mathcal{S}_{M}^{E}(U)$ is a closed embedding and res ${ }_{U, V}: \mathcal{G}_{M}^{E}(U) \rightarrow \mathcal{G}_{M}^{E}(V)$ is onto.

Proof. The map ext $t_{V, U}$ is a closed embedding by the theorem. Hence by the Hahn Banach theorem the dual map $\operatorname{res}_{U, V}$ is onto.

\subsubsection{Remarks}

Remark 5.4.5 Some of the ideas of the proof of theorem 5.4.1 are taken from Casselman's unpublished work [Cas2].

Remark 5.4.6 Since extension by zero $\mathcal{S}(U) \cong W_{Z}$ is a continuous linear isomorphism, the inverse map is also continuous by Banach open map theorem. In fact, as it often happens, our proof that extension is onto can be easily refined to prove that the inverse map is continuous. 


\subsection{Generalized Schwartz sections supported on closed sub- manifolds}

Definition 5.5.1 Let $M$ be a restricted topological space, and $F$ be a sheaf on $M$. Let $Z \subset M$ be a closed subset. A global section of $F$ is said to be supported in $Z$ if its restriction to the complement of $Z$ is zero.

Remark 5.5.1 Unfortunately, if we try to define support of a section, it will not be a closed set in general, since infinite intersection of closed sets in the restricted topology does not have to be closed. Also, we cannot in general consider its closure, because there is no closure in restricted topology by the same reason.

Proposition 5.5.2 Let $E \rightarrow M$ be a Nash bundle and $Z \subset M$ be a closed (semialgebraic) subset. Let $\xi \in \mathcal{G}_{M}^{E}(M)$. Then $\xi$ is supported in $Z$ if and only if for any $\phi \in \mathcal{S}_{M}^{\widetilde{E}}(M)$ which vanishes with all its derivatives on $Z, \xi(\phi)=0$.

Proof. It is an immediate corollary of the characterization of Schwartz sections on an open subset (theorem 5.4.3).

Lemma 5.5.3 Let $M$ be a Nash manifold and let $Z \subset M$ be a closed Nash submanifold. Let $\Delta$ be the map

$$
\{\phi \in \mathcal{S}(M) \mid \phi \text { is } 0 \text { on } Z \text { with first } i-1 \text { derivatives }\} \rightarrow \mathcal{S}\left(Z, S^{i}\left(C N_{Z}^{M}\right)\right) \text {, }
$$

given by the $i$-th derivative, where $S^{i}$ means $i$-th symmetric power and $C N_{Z}^{M}$ is the conormal bundle. Then $\Delta$ is well defined and onto.

Proof. For $M=Z \times \mathbb{R}^{d}$ the lemma is trivial. For the general case, it is proved in the same way as theorem 4.6.1 - using Nash tubular neighborhood.

Corollary 5.5.4 Property $\square$ holds. Namely, let $Z \subset M$ be a closed Nash subset. Consider $V=\{\xi \in \mathcal{G}(M, E) \mid \xi$ is supported in $Z\}$. It has a canonical filtration $V_{i}$ such that its factors are canonically isomorphic to $\mathcal{G}\left(Z,\left.\left.E\right|_{Z} \otimes S^{i}\left(N_{Z}^{M}\right) \otimes D_{M}^{*}\right|_{Z} \otimes D_{Z}\right)$ where $N_{Z}^{M}$ is the normal bundle.

Proof. Denote $K:=\mathcal{S}_{M}^{\widetilde{E}}(M), L:=\mathcal{S}_{M}^{\widetilde{E}}(U)$. Then $V=(K / L)^{*}$. Define $K_{i}:=$ $\left\{\phi \in K \mid\right.$ for any Nash differential operator $D$ of degree $\left.\leq i-1,\left.D \phi\right|_{Z}=0\right\} . K_{0}=K$ and $\bigcap K_{i}=L$ and by the previous lemma $K_{i} / K_{i+1}=\mathcal{S}\left(Z,\left.\widetilde{E}\right|_{Z} \otimes S^{i}\left(C N_{Z}^{M}\right)\right)$. Define $V_{i}=\left(K / K_{i}\right)^{*}$. It is the requested filtration.

This corollary appeared in a similar form in Casselman's unpublished work [Cas2] and in another similar form in [CHM]. 


\section{A Appendix}

\section{A.1 Nash structures on standard bundles}

In this section we construct Nash structures on standard bundles from differential geometry.

Theorem A.1.1 The tangent bundle of any Nash manifold has a canonical structure of a Nash bundle.

Corollary A.1.2 Tangent, normal and conormal bundles, the bundle of differential $k$-forms, etc., of a Nash manifold have canonical structure of Nash bundles.

Proof of the theorem. In order to prove this theorem we have to prove two statements:

1. The total space of the tangent bundle has a canonical structure of a Nash manifold.

2. There is a finite cover of $M$ by Nash trivializations of the tangent bundle.

Proof of 1. It is enough to prove the proposition for a closed affine Nash submanifold $M \subset \mathbb{R}^{n}$. In this case, the tangent bundle of $M$ is defined in $\mathbb{R}^{2 n}$ by semi-algebraic conditions. Hence, by the Tarski-Seidenberg principle it is a closed affine Nash submanifold of $\mathbb{R}^{2 n}$. So the proposition holds true for the tangent bundle, and hence also for normal and conormal bundles, the bundle of differential $k$-forms, etc.

Proof of 2. It is sufficient to prove for the cotangent bundle instead of the tangent one. Also, we can suppose that the manifold is affine.

Let $M \subset \mathbb{R}^{n}$ be a closed Nash embedding. We have a map $\pi$ from $\left(\mathbb{R}^{n}\right)^{*}$ to covector fields on $M$. Let $\left\{e_{i}\right\}$ be a basis of $\left(\mathbb{R}^{n}\right)^{*}, d:=\operatorname{dim} M$. For any subset $S \subset\{1, \ldots, n\}$ of cardinality $d$ we define $f_{S}:=\operatorname{det}\left(\pi\left(e_{i}\right), i \in S\right)$. Let $U_{S}:=\left\{x \in M \mid f_{S}(x) \neq 0\right\}$. Since $\pi$ is fiberwise onto, we know from linear algebra that $U_{S}$ cover $M$. Clearly, every $U_{S}$ is an open semi-algebraic subset and the cotangent bundle is trivializable on it.

Proposition A.1.3 Let $M \subset \mathbb{R}^{n}$ be a closed Nash submanifold. Then the space of Nash covector fields on $M$ is generated over $\mathcal{N}(M)$ by the covector fields $d x_{i}$. theorem.

Proof. The fields $d x_{i}$ are exactly the fields $\pi\left(e_{i}\right)$ from the proof of the last

\section{A.1.1 The bundle of densities $D_{M}$}

Now we wish to define structure of Nash bundle on the bundle of densities. It is done in the same way as in differential geometry. 
We remind that the $\mathbb{Z} / 2 \mathbb{Z}$ - torsor Orient $_{M}$ of orientations is defined as the quotient of the bundle $\Omega_{M}^{\text {top }}$ of top differential forms by the multiplicative action of $\mathbb{R}_{>0}$. The standard correspondence between torsors and bundles gives us a bundle of orientations $B O r=$ Orient $_{M} \bigotimes_{\mathbb{Z} / 2 \mathbb{Z}} \mathbb{R}$.

The bundle of densities $D_{M}$ is defined as the tensor product of $B O r_{M}$ and $\Omega_{M}^{\text {top }}$, and its global smooth sections are smooth measures on $M$.

Definition A.1.1 Let $M$ be a Nash manifold. We would like to define a Nash structure on the bundle BOr ${ }_{M}$ of orientations on $M$. Consider the $\mathbb{Z} / 2 \mathbb{Z}$ torsor Orient $_{M}$ of orientations on $M$. Since $\Omega_{M}^{\text {top }}$ is a Nash bundle, there exists a finite cover of $M$ by open (semi-algebraic) subsets $M=\bigcup U_{i}$ such that Orient $\left._{M}\right|_{U_{i}}$ is isomorphic to $\mathbb{Z} / 2 \mathbb{Z} \times U_{i}$ as a smooth locally trivial fibration. Choose such isomorphisms $\beta_{i}$. These $\beta_{i}$ define the structure of a Nash locally trivial fibration on Orient $_{M}$. It does not depend on the choice of $\beta_{i}$ since the fiber is finite. We define the bundle $\mathrm{BOr}_{M}$ of orientations on $M$ to be the bundle corresponding to the $\mathbb{Z} / 2 \mathbb{Z}$-torsor Orient $_{M}$ and the sign representation of $\mathbb{Z} / 2 \mathbb{Z}$. In other words, BOr $_{M}=\left(\right.$ Orient $\left._{M} \times \mathbb{R}\right) /(\mathbb{Z} / 2 \mathbb{Z})$ where $\mathbb{Z} / 2 \mathbb{Z}$ acts diagonally, and on $\mathbb{R}$ it acts by sign. It has an obvious structure of a Nash bundle.

Definition A.1.2 We define the bundle $D_{M}$ of densities on $M$ by $D_{M}=$ $\operatorname{BOr}_{M} \bigotimes_{M} \Omega_{M}^{t o p}$

\section{A.2 Semi-algebraic notions on Nash manifolds}

Definition A.2.1 A subset $A$ of an affine Nash manifold $M \subset \mathbb{R}^{n}$ is called semialgebraic iff it is semi-algebraic in $\mathbb{R}^{n}$. Clearly this notion does not depend on the embedding $M \hookrightarrow \mathbb{R}^{n}$.

A subset $A$ of a Nash manifold $M$ is called semi-algebraic iff its intersection with any open affine Nash submanifold is semi-algebraic .

A map $\nu$ between Nash manifolds $M$ and $N$ is called semi-algebraic iff its graph is a semi-algebraic subset of $M \times N$.

We will need the following technical lemma, which is a direct consequence of lemma 2.2.11 about majoration of semi-algebraic functions by polynomials.

Lemma A.2.1 Let $M$ be an affine Nash manifold. Then any continuous semialgebraic function on it can be majorated by a Nash function, and any continuous strictly positive semi-algebraic function on it can be bounded from below by a strictly positive Nash function. 
Remark A.2.2 Let $\phi: M \rightarrow N$ be a semi-algebraic map between Nash manifolds. Clearly, $\phi$ is continuous as a map of classical topological spaces if and only if $\phi$ is continuous as a map of restricted topological spaces.

Theorem A.2.3 Let $M$ be a Nash manifold. Then there exists a semi-algebraic continuous embedding $\nu: M \hookrightarrow \mathbb{R}^{n}$, that means a semi-algebraic map $\nu: M \rightarrow \mathbb{R}^{n}$ which is a homeomorphism to its image.

For proof see [Shi], page 142, theorem III.1.1 .

Corollary A.2.4 Let $M$ be a Nash manifold. Then there exists a semi-algebraic continuous metric $d: M \times M \rightarrow \mathbb{R}$.

Remark A.2.5 This corollary can be proven directly by defining the metric for open affine Nash subsets that cover $M$ and then gluing.

Proposition A.2.6 Let $U$ be an open (semi-algebraic) subset of a Nash manifold M. Then its closure $\bar{U}$ in the usual topology is closed semi-algebraic, that is its complement $\bar{U}^{c}$ is open semi-algebraic.

Proof. It is enough to prove for affine $M$. This case easily follows from TarskiSeidenberg principle.

Remark A.2.7 The last proposition shows that open sets have closure in the restricted topology.

\section{A.3 Covers}

In this section we give some definitions and propositions that help us to work with covers. The propositions of this subsection are versions of a general statement which says that any open cover of a Nash manifold can be replaced by a finer cover that has some nice properties. This subsection is used in the proofs of partition of unity both for affine and general Nash manifolds (sections 4.4 and 5.2). The central statement of this section is proposition A.3.4.

Notation A.3.1 Let $M$ be a Nash manifold and $F$ be a continuous semi-algebraic function on $M$. We denote $M_{F}:=\{x \in M \mid F(x) \neq 0\}$.

Definition A.3.2 Let $M$ be a Nash manifold. A continuous semi-algebraic function $F$ on $M$ is called basic if $\left.F\right|_{M_{F}}$ is a positive Nash function.

The the above definition is motivated by the following lemma. 
Lemma A.3.1 Let $M$ be an affine Nash manifold. Then it has a basis of open sets of the form $M_{F}$ where $F$ is a basic function.

This lemma follows directly from the finiteness theorem (2.3.2).

Definition A.3.3 We say that a cover $M=\bigcup_{j=1}^{m} V_{j}$ is a refinement of the cover $M=\bigcup_{i=1}^{n} U_{i}$ if for any $j$ there exists $i$ such that $V_{j} \subset U_{i}$.

We say that a cover $M=\bigcup_{j=1}^{m} V_{j}$ is a proper refinement of the cover $M=\bigcup_{i=1}^{n} U_{i}$ if for any $j$ there exists $i$ such that $\overline{V_{j}} \subset U_{i}$.

Proposition A.3.2 Let $M=\bigcup_{i=1}^{n} U_{i}$ be a finite open (semi-algebraic) cover of an affine Nash manifold $M$ such that $U_{i}=\left\{x \in M \mid F_{i j}(x)>0\right.$ for $\left.j=1 \ldots n_{i}\right\}$ for certain continuous semi-algebraic functions $F_{i j}: M \rightarrow \mathbb{R}$. Then there exists a strictly positive Nash function $g$ such that the sets $V_{i}:=\left\{x \in M \mid F_{i j}(x)>g(x)\right.$ for $\left.j=1 \ldots n_{i}\right\}$ also cover $M$.

Proof.

Denote $G_{i j}:=\max \left(F_{i j}, 0\right), G^{\prime}:=\frac{1}{2} \max _{i=1}^{n} \min _{j=1}^{n} G_{i j}$. By lemma A.2.1 there exists a Nash function $g$ such that $0<g \leq G^{\prime}$.

Proposition A.3.3 Let $M=\bigcup_{i=1}^{n} U_{i}$ be a finite open (semi-algebraic) cover of a Nash manifold $M$. Then there exists a finite open (semi-algebraic) cover $M=\bigcup_{i=1}^{n} V_{i}$ which is a proper refinement of $\left\{U_{i}\right\}$.

Proof. Let $d$ be the metric from corollary A.2.4. If a set $A$ is closed in the classical topology, then the distance $d(x, A):=\inf _{y \in A} d(x, y)$ is strictly positive for all points $x$ outside $A$. Now define $F_{i}: M \rightarrow \mathbb{R}$ by $F_{i}(x)=d\left(x, M \backslash U_{i}\right)$. It is semi-algebraic by the Tarski-Seidenberg principle. Define $G=\left(\sum_{i=1}^{n} F_{i}\right) / 2 n$ and $V_{i}=\left\{x \in M \mid \quad F_{i}(x)>\right.$ $G(x)\}$. It is easy to see that $V_{i}$ is a proper refinement of $U_{i}$.

In order to formulate the central proposition of this section, we need to define one technical notion.

Definition A.3.4 A collection of continuous semi-algebraic functions $\left\{F_{i}\right\}$ is called basic collection if every one of them is basic, and in every point of $M$ one of them is larger than 1. 
Proposition A.3.4 Let $M=\bigcup_{i=1}^{n} U_{i}$ be a finite open (semi-algebraic) cover of a Nash manifold $M$. Then there exists a basic collection of continuous semi-algebraic functions $F_{j}$ on $M$ such that the cover $M_{F_{j}}$ is a refinement of $\left\{U_{i}\right\}$.

Proof. Cover $U_{i}$ by affine open subsets $V_{i j}$. By proposition A.3.3 there exist $V_{i j}^{\prime} \subset \overline{V_{i j}^{\prime}} \subset V_{i j}$ which also cover $M$. By proposition A.3.1 which follows from the finiteness theorem, there exist functions $G_{i j k}: V_{i j} \rightarrow \mathbb{R}$ and open sets $V_{i j k}^{\prime}$ such that $V_{i j k}^{\prime}=\left\{x \in V_{i j} \mid G_{i j k}(x) \neq 0\right\},\left.G_{i j k}\right|_{V_{i j k}^{\prime}}$ is positive and Nash and $\bigcup_{k} V_{i j k}^{\prime}=V_{i j}^{\prime}$. In order to have a unified system of indexes we denote $V_{i j k}:=V_{i j}$. It gives a finite cover of $M$ which is a refinement of $U_{i}$, we re-index it to one index cover $V_{l}$. By the same re-indexation we define $G_{l}$ and $V_{l}^{\prime}$. Extend $G_{l}$ by zero to a function $\widetilde{G}_{l}$ on $M$. It is continuous. Denote $G=\left(\sum \widetilde{G_{l}}\right) /(2 n)$ where $n$ is the number of values of the index l. Consider $\left.G\right|_{V_{l}}$. This is a strictly positive continuous semi-algebraic function on an affine Nash manifold, hence by proposition A.2.1 it can be bounded from below by a strictly positive Nash function $g_{l}^{\prime}$. Denote $H_{l}:=G_{l} / g_{l}^{\prime}$. Extending $H_{l}$ by zero outside $V_{l}$ we obtain a collection of continuous semi-algebraic functions $F_{l}$ to $M$. It is easy to see that $\left\{F_{l}\right\}$ is a basic collection and $M_{F_{l}}$ is a refinement of $U_{i}$.

Remark A.3.5 We do not know whether the sets $M_{F}$, where $F$ is a basic function, form a basis of the restricted topology for a non-affine Nash manifold M. May be this statement is difficult to verify for the following reason: the notion of basis is not an appropriate notion for restricted topology. The property we have just proven is slightly weaker, but enough for our purposes and probably can be formulated for any Grothendieck site, unlike the usual notion of basis.

\section{A.4 Cosheaves over restricted topological spaces}

Since Schwartz functions cannot be restricted to open subsets, but can be extended by 0 from open subsets, we need the notions of a pre-cosheaf and a cosheaf. There are such notions for any Grothendieck site (namely, a pre-sheaf and a sheaf with values in the opposite category). We will now repeat their definitions for restricted topology.

Definition A.4.1 A pre-cosheaf $F$ on a restricted topological space $M$ is a covariant functor from the category (Top $(M)$ which has open sets as its objects and inclusions as morphisms) to the category of abelian groups, vector spaces, etc.

In other words, it is an assignment $U \mapsto F(U)$ for every open $U$ of an abelian group, vector space, etc., and for every inclusion of open sets $V \subset U$ an extension morphism ext $_{V, U}: F(V) \rightarrow F(U)$ which satisfy: ext $t_{U, U}=I d$ and for $W \subset V \subset U$, $\operatorname{ext}_{V, U} \circ \operatorname{ext}_{W, V}=\operatorname{ext}_{W, U}$. 
Definition A.4.2 A cosheaf $F$ on a restricted topological space $M$ is a pre-cosheaf on $M$ fulfilling the conditions dual to the usual sheaf conditions, and with only finite open covers allowed. This means: for any open set $U$ and any finite cover $U_{i}$ of $M$ by open subsets, the sequence

$$
\bigoplus_{i=1}^{n-1} \bigoplus_{j=i+1}^{n} F\left(U_{i} \cap U_{j}\right) \rightarrow \bigoplus_{i=1}^{n} F\left(U_{i}\right) \rightarrow F(U) \rightarrow 0
$$

is exact.

Here, the first map is defined by

$$
\bigoplus_{i=1}^{n-1} \bigoplus_{j=i+1}^{n} \xi_{i j} \mapsto \sum_{i=1}^{n-1} \sum_{j=i+1}^{n} \operatorname{ext}_{U_{i} \cap U_{j}, U_{i}}\left(\xi_{i j}\right)-\operatorname{ext}_{U_{i} \cap U_{j}, U_{j}}\left(\xi_{i j}\right)
$$

and the second one by

$$
\bigoplus_{i=1}^{n} \xi_{i} \mapsto \sum_{i=1}^{n} \operatorname{ext}_{U_{i}, U}\left(\xi_{i}\right)
$$

\section{References}

[AGS] Avraham Aizenbud, Dmitry Gourevitch, Eitan Sayag: $G L(n+1, F), G L(n, F))$ is a Gelfand pair for any local field F. arXiv:0709.1273v2

[BCR] Bochnak, J; Coste, M; Roy, M-F: Real Algebraic Geometry (1998)

[Cas1] Casselman, W: Introduction to the Schwartz Space of $\Gamma \backslash G$. Canadian Journal Mathematics Vol.XL, No2, 285-320 (1989)

[Cas2] Casselman,W: Bruhat Filtration. Unpublished

[CHM] Casselman, William; Hecht, Henryk; Miličić, Dragan: Bruhat filtrations and Whittaker vectors for real groups. The mathematical legacy of Harish-Chandra (Baltimore, MD, 1998), 151-190, Proc. Sympos. Pure Math., 68, Amer. Math. Soc., Providence, RI, (2000)

$[\mathrm{dCl}] \mathrm{du}$ Cloux, Fokko: Sur les reprsentations diffrentiables des groupes de Lie algbriques. Annales scientifiques de l.N.S. 4e srie, tome 24, no 3, p. 257-318 (1991).

[DK] Delfs, H; Knebush, M: Semialgebraic Topology Over a Real Closed Field II. Mathematische Zeitschrift 178, 175-213 (1981) 
[HC] Harish-Chandra: Automorphic Forms on Semi-Simple Lie Groups. Bulletin of American Mathematical Society 76, 529-551 (1970)

[KS] Kashiwara, M.; Schapira, P.: Ind-Sheaves. Asterisques 271, (2001)

[Mal] Malgrange, B: Ideals of Differentiable Functions, Oxford University Press (1966)

[Mor] Morando, G.:An existence theorem for tempered solutions of D-modules on complex curves, arXiv: math.AG/0505498 (2006)

[Pre] Prelli, L.: Sheaves on subanalytic sites, arXiv: math.AG/0505498 (2007)

[Shi] Shiota, M: Nash Manifolds. Lecture Notes in Mathematics 1269 (1987) 